\title{
La notion de tiers-espace et la traduction juridique : quelle articulation?
}

\author{
Margarete Flöter-Durr ${ }^{1}$ (D) $\cdot$ Paulina Nowak-Korcz ${ }^{2}$ (D)
}

Accepted: 29 September 2020 / Published online: 13 October 2020

(c) The Author(s) 2020

\begin{abstract}
In recent years, we have noticed in many research areas a growing interest in the translation paradigm. In this article we discuss the theory of "cultural translation", developed by Homi Bhabha in the context of postcolonial studies, and his concept of "Third Space" (2007). This theory aims to describe the different processes at play in the creation of identity within a space in which several cultures coexist. According to Bhabha, "the Third Space, though unrepresentable in itself, constitutes the discursive conditions of enunciation that ensure that the meaning and symbols of culture have no primordial unity or fixity; even the same signs can be appropriated, translated, rehistorised and read anew". In this article, we aim to examine to what extent this theory can be applied to translation, especially to legal translation. The concepts of "Third Space" and hybridity developed by Bhabha undoubtedly have a certain appeal for the translator. However, from the methodological point of view other approaches seem to allow for a better understanding of the translation aspects raised by Bhabha in his theory. We therefore provide an interesting analysis of the "Third Space" based on the concept of interval by Cassin (Éloge de la traduction. Compliquer l'universel, Fayard, Paris, 2016), which is defined as a complex zone of interactions and interferences. This analysis is completed by examining the concept of frontiers by Moréteau (Revue internationale de droit comparé 4(61):695-713, 2009. DOI: 10.3406/ridc.2009.19911) which is implicitly present in the concept of interval. In order to analyse the concept of hybridity, the methodological framework of transdifference developed by Srubar (Kultur und Semantik, VS Verlag für Sozialwissenschaften, Wiesbaden, 2009) can also be used.
\end{abstract}

Keywords Third Space $\cdot$ Hybridity $\cdot$ Legal translation $\cdot$ Cultural translation

Paulina Nowak-Korcz

paulina.nowak-korcz@uni.lodz.pl

Margarete Flöter-Durr

info@mg-traductions.eu

1 UR 1339, Membre associé LiLPa, Université de Strasbourg, Strasbourg, France

2 Département des langues spécialisées et de la communication interculturelle, Faculté de Philologie, Université de Lodz, Lodz, Poland 


\section{Résumé}

Ces dernières années, le paradigme de la traduction semble gagner en attractivité dans de nombreux domaines de recherche. Dans le présent article, nous abordons la théorie de la «traduction culturelle » élaborée par Homi Bhabha dans le contexte d'études postcoloniales et sa notion de «tiers espace » (2007). Cette théorie vise à rendre compte des processus de production de l'identité en présence de plusieurs cultures. Selon Bhabha, « le tiers-espace, quoique irreprésentable en soi, constitue les conditions discursives de l'énonciation qui attestent que le sens et les symboles culturels n'ont pas d'unité ou de fixité primordiale et que les mêmes signes peuvent être appropriés, traduits, rehistoricisés et réinterprétés ». La question qui se pose est de savoir dans quelle mesure cette théorie est susceptible d'être transposée à la traduction, et plus spécialement à la traduction juridique. Les notions de «tiers-espace » et d'hybridité élaborées par Bhabha possèdent indéniablement un certain potentiel d'attractivité pour le traducteur. Méthodologiquement, il nous semble toutefois que d'autres approches sont mieux à même d'en rendre compte. Ainsi, une articulation intéressante du «tiers-espace » peut être proposée à partir de la notion d'intervalle, conçue comme une zone complexe d'interactions et d'interférences, élaborée par Cassin (2016). Cette articulation peut être complétée en mobilisant la notion de frontière (Moréteau 2009), présente implicitement dans celle d'intervalle. Pour articuler la notion d'hybridité, il est également possible de mobiliser le cadre méthodologique de la transdifférence proposé par Srubar (2009).

Mots-clés Tiers-espace $\cdot$ Hybridité $\cdot$ Traduction juridique $\cdot$ Traduction culturelle

\section{Introduction}

Dans la conception classique de Jakobson, la traduction - opérateur de la diversité s'il en est [47, p. 263] - revêt trois formes distinctes : la traduction intralinguale ou reformulation, la traduction interlinguale ou traduction proprement dite et la traduction intersémiotique ou «transmutation », c'est-à-dire l'interprétation des signes linguistiques au moyen de systèmes de signes non linguistiques [23, p. 233]. Cette typologie a fait l'objet de nombreuses critiques ${ }^{1}$ en raison, notamment du sens restreint conféré par Jakobson au terme de «traduction ». En effet, à partir d'une définition large du signe et de la distinction entre signes tangibles et intangibles [24, p. 37], le sens du terme «traduction » peut être élargi jusqu'à recouvrir les transformations sémiotiques entre les différents types de signes [24, p. 33] au sein d'une ou plusieurs sémiosphères ${ }^{2}$. Le terme de traduction recèle ainsi un fort potentiel

\footnotetext{
${ }^{1}$ Sur la critique de la triade jakobsonienne par Eco, Gorlée et Toury, voir Jia [24].

${ }^{2}$ Le concept de « sémiosphère » a été introduit par Lotman, Yuri, M. [27]. Il est défini comme «un espace ou un medium servant de support aux signes d'une culture donnée où ont lieu les activités ou transformations sémiotiques. (Notre traduction du passage original « (...) a space or medium carrying the signs of a give culture and the place in which semiotic activities or transformations occur' », cf. Jia [24].
} 
d'extension qui permet de l'investir de nouvelles significations et d'étendre de plus en plus ses limites. En outre, l'intrication entre langue et culture définie comme « un système de significations » [21, p. 66] et l'avènement d'un monde multipolaire [60] dans le sillage de la mondialisation confèrent au phénomène traductif une ampleur sans précédent. Tous ces éléments participent d'une attractivité indéniable du paradigme traductif pour un certain nombre de disciplines scientifiques autres que la linguistique ou la traductologie. Ainsi, par exemple, en droit, François Ost cherche à étendre le paradigme traductif à l'ensemble de la science juridique [31, p. 9] tandis que pour Benoît Frydman, la traduction joue un rôle majeur en tant qu'opérateur du dialogue des cultures juridiques visant une harmonisation progressive des règles de droit et des ordres juridiques dans le processus de la mondialisation [17, p. 16]. En sociologie, Joachim Renn propose d'utiliser la traduction pour modéliser les relations entre les « entités d'intégration » sous réserve toutefois de concevoir la traduction non pas comme un transfert de signification par-delà les frontières linguistiques ou comme une recherche d'équivalents, mais comme une pratique de dépassement des frontières [34, p. 157]. Le même attrait pour le paradigme traductif s'observe également dans le domaine de Cultural Studies ${ }^{3}$. Ainsi, Doris BachmannMedick, plaide en faveur d'un élargissement du concept de la traduction susceptible d'entraîner un « tournant translationnel » en sciences humaines et sociales. Ce tournant a vocation à prolonger un certain nombre d'autres tournants qui se sont produits dans ces domaines, à savoir le tournant interprétatif, performatif, postcolonial, etc. [3, p. 186]. Par ailleurs, elle propose d'adopter le paradigme traductif comme une catégorie analytique permettant de rendre compte des transferts interculturels et des différences et tensions qui existent entre les différentes disciplines scientifiques et écoles de pensée [2, p. 187]. C'est dans ce contexte plus large que s'inscrit la théorie élaborée par Homi Bhabha dans le cadre des études postcoloniales ${ }^{4}$ et ses notions de traduction culturelle, de tiers-espace et d'hybridité [7]. Ces notions ont connu une carrière fulgurante et ont été reprises dans de nombreux domaines, jusqu'à dans le marketing [53]. Simultanément, elles ont suscité une vive controverse et ont fait l'objet de critique non moins importante [46]. En ce qui concerne la traduction interlinguale, notamment juridique, elles exercent indéniablement un attrait sur les travaux théoriques [52]. Le présent article vise à montrer qu'en ce qui concerne la traduction interlinguale en général, et la traduction juridique en particulier, l'opérationnalisation des notions conceptualisées par Bhabha semble difficile en raison des difficultés qu'elle soulève sur le plan théorique et pratique. Ces difficultés procèdent de plusieurs éléments, à savoir : une conception trop large de la traduction qui mêle les aspects linguistiques et sémiotiques, la confusion entre l'altérité (das Fremde) et l'étrangeté (die Fremdheit) et, enfin, la nature agonistique

\footnotetext{
${ }^{3}$ Nous nous conformons à l'usage généralement accepté en français et conservons le vocable anglosaxon, dans la mesure où il n'existe pas de véritable ,équivalent à cette entité épistémologique dans la conscience épistémologique française”, cf. Chalard-Fillaudeau, Anne, Raulet, Gérard [11].

4 Il s'agit d'un courant apparu dans les années $80 \mathrm{du} \mathrm{XX}^{\mathrm{ème}}$ siècle dans les universités américaines, diffusé par la suite en Europe. Ce nouveau champ d'études suscite une importante controverse pour des raisons diverses. Sur ce point, voir notamment Bayart [6].
} 
de la traduction qui, dans un processus d'hybridation à l'infini, fait obstacle à l'intégration de l'altérité et à la production du sens. Dans cette perspective, la traduction ne peut que manquer à réaliser son objectif premier qui consiste à fixer le sens et à permettre la compréhension réciproque, et donc la communication. Nonobstant ces difficultés, les concepts de tiers-espace et d'hybridité possèdent indéniablement un certain potentiel d'attractivité pour le traducteur compte tenu d'une certaine similitude des processus de la traduction culturelle et interlinguistique. En effet, en traduction interlinguistique il se crée temporairement un espace où le texte en langue source est resémantisé afin de lui conférer un sens en langue cible. Toutefois, pour rendre compte des phénomènes de tiers-espace et d'hybridité en traduction interlinguale, on peut recourir à d'autres outils descriptifs permettant de les caractériser. C'est ce que nous proposons dans le présent article qui se structure en trois parties. La première partie est consacrée à exposer les principales notions de la théorie de Bhabha. Elle est précédée d'un certain nombre d'observations liminaires permettant de préciser le type de traduction envisagée ici et de problématiser succinctement les risques liés à la migration des concepts entre les disciplines scientifiques et l'usage des métaphores conceptuelles. La seconde partie est consacrée à la critique des notions de Bhabha du point de vue de la traduction interlinguale. Enfin, la troisième partie est consacrée à montrer que nonobstant les processus d'hybridité qui peuvent être observés du fait de la présence simultanée des sémantiques distinctes en traduction interlinguale, celle-ci constitue le lieu d'intégration de la diversité et de l'altérité. Méthodologiquement, nous mobiliserons les concepts aptes à décrire le lieu de cette intégration et à rendre compte des mécanismes linguistiques d'intégration et de la production du sens. En effet, et contrairement à l' affirmation de Bhabha selon laquelle un symbole univoque est transcodé dans le tiers-espace en des signes polysémiques de manière à rendre impossible toute fixation du sens [46, p . 124], la traduction interlinguale ne peut se passer de fixer le sens, fût-ce de manière imparfaite et provisoire.

Pour caractériser les processus d'hybridité à l'œuvre dans la traduction interlinguale, la notion de transdifférence sera mobilisée [44]. Elle permet d'appréhender la constitution du sens en présence de sémantiques distinctes.

Pour préciser la notion de tiers-espace en traduction interlinguale, nous recourons aux notions d'intervalle de Cassin [10] et « d'entre-deux » de Wissmann [56]. Elles déterminent la position du traducteur en tant que médiateur entre les univers linguistiques, culturels et juridiques distincts et en tant que l'opérateur du passage de l'un à l'autre. La notion de frontière [29], présente implicitement dans celle d'intervalle complète cette caractérisation du tiers-espace en traduction interlinguale. Pour caractériser le mécanisme de l'ouverture des frontières entre les espaces circonscrits par les différentes langues et cultures, la notion de pertinence d'extraction schützéenne [38], non mentaliste, sera mobilisée. En ce qui concerne la traduction interlinguale, elle présente un double intérêt : elle permet de caractériser la production du sens en termes de sélection et de combinaison d'éléments lexicaux, syntaxiques et stylistiques, d'une part, et d'autre part, elle permet de montrer que le phénomène d'altérité est omniprésent et qu'une exposition prolongée d'un individu étranger à l'interaction avec un groupe d'accueil déclenche les mécanismes d'intégration sociale [40]. Nous espérons que nos développements permettront de montrer que 
la traduction pragmatique interlinguistique et interculturelle est avant tout un lieu d'intégration de l'altérité. Irréductiblement imparfaite, la traduction remplit néanmoins cette fonction de l'hospitalité langagière qui permet «d'accueillir l'autre en tant qu'autre » [32, p. 15] sans le faire disparaître derrière l'écran de l'étrangeté.

\section{Les notions clés de la théorie d'Homi Bhabha}

Homi Bhabha est un théoricien de la littérature et de la culture postcoloniales. La théorie qu'il expose dans un ensemble de textes réunis dans l'ouvrage intitulé en français Les lieux de la culture [7] est construite dans une approche transdisciplinaire et poststructuraliste [46, p. 37]. L'éclectisme méthodologique permet à Bhabha de nourrir sa réflexion des apports théoriques aussi divers que la psychoanalyse de Freud et de Lacan, la théorie du roman et le concept de polyphonie de Bakhtin, ou la philosophie de Walter Benjamin, convoquée à titre de caution pour sa conception singulière de la traduction culturelle. La théorie de Bhabha est une tentative de repenser les structures coloniales du pouvoir, les formes de représentations culturelles et la dynamique des contacts culturels. Sa pensée se caractérise par une intrication étroite des éléments issus de la littérature, de l'art et de la culture moderne [46, p. 10]. Pour construire sa théorie, Bhabha opère essentiellement avec les notions clés telles que la différence culturelle définie comme une forme d'antagonisme social [7, p. 289], l'hybridité, le tiers espace et la traduction culturelle. Toutes ces notions s'inscrivent dans une conception spécifique de la culture définie comme « une production inégale et inachevée de la signification et de la valeur » $[46, \text { p. } 37]^{5}$. En ce qui concerne la notion de culture, il est important de souligner qu'elle se fonde sur l'opposition entre différence et diversité culturelle [8, p. 96]. Selon Bhabha, seule la prise en compte de la différence culturelle permet d'appréhender la culture dans la plénitude de son acception tandis que la diversité culturelle conduit la culture dominante à imposer « une norme transparente » qui aboutit à endiguer la différence culturelle [8, p. 96]. Il s'agit d'une vision singulière de la culture, dans la mesure où elle passe outre le fait que l'altérité entendue comme une « expérience différente du monde de la vie » est un processus de transformation dans la durée qui ouvre la voie à l'intégration de l'étranger dans un groupe d'accueil [40, p. 22]. Or dans sa conception de la culture, Bhabha réserve, semble-t-il, peu de place à l'intégration. Au contraire, il souligne l'éclatement des identités et des cultures en une multitude d'éléments hétérogènes dans les processus d'hybridation au sein de ce qu'il désigne comme le tiers-espace. Bhabha souligne le caractère construit de toute culture et sa nature discursive [46, p. 59] ce qui - soit dit en passant - revient à affirmer une banalité, du moins du point de vue des récents acquis de la théorie de la traduction juridique [9, 18]. Rejetant la conception holistique de la culture, Bhabha la conçoit comme une entité instable, hétérogène et variable, caractérisée par la différence et l'ambivalence [46, pp. 41-42] et l'inscrit ainsi dans le paradigme constructiviste. À partir de cette conception de la culture, il conceptualise

\footnotetext{
${ }_{5}^{5}$ Nous citons d'après Struve : „eine ungleichmäßige, unvollendete Produktion von Bedeutung und Wert“.
} 
les notions piliers de sa théorie : la traduction culturelle entendue comme « le lieu de lutte au centre de la représentation coloniale » [46, p. 37], l'hybridité et le tiersespace. Pour les besoins de notre exposé, nous nous intéresserons exclusivement à ces trois notions, et ce uniquement pour autant qu'elles sont pertinentes pour notre propos.

Avant d'exposer les principales notions de la théorie de la traduction culturelle, il nous semble opportun de formuler un certain nombre d'observations liminaires en ce qui concerne le type de traduction envisagé dans le présent article, l'emprunt des concepts issus des autres disciplines scientifiques et l'usage des métaphores conceptuelles. En effet, tant le recours à l'emprunt que l'usage des métaphores conceptuelles comportent un certain nombre de risques.

\subsection{Les observations liminaires}

\subsubsection{Sur la nature pragmatique de la traduction juridique}

Dans le vaste champ de la traduction interlinguale, nous prenons pour objet la traduction dite pragmatique [16], appelée aussi traduction professionnelle ou spécialisée [25, p. 628], qui revêt une dimension pragmatique à plusieurs titres. En premier lieu, elle est pragmatique au sens large, dans la mesure où elle constitue un moyen permettant d'atteindre un objectif [55, p. 463]. En second lieu, elle est pragmatique, dans la mesure où elle participe d'une praxis sociale et qu'elle constitue une pratique [12, p. 38]. En tant que pratique, la traduction vise une tâche concrète et implique systématiquement un choix, et donc une décision. La décision du sens est donc immanente à toute traduction pragmatique. En troisième lieu, la traduction est pragmatique, dans la mesure où elle répond à une visée communicative et consiste à produire des textes pourvus de sens [16, p. 231]. La traduction juridique en tant qu'acte de communication en droit est constitutive d'une pratique sociale et relève à ce titre de la traduction pragmatique. En outre, la traduction juridique peut être qualifiée de pragmatique, dans la mesure où elle constitue une zone de contact épistémologique où l'on observe une certaine hybridation du discours des sciences exactes et des sciences humaines à laquelle correspond une hybridation de genres. La traduction pragmatique ainsi définie se distingue de la traduction littéraire. Celleci est écartée dans le présent article, dans la mesure où la question de l'hybridité s'y pose différemment, car le choix du mode d'écriture et l'hybridation générique et linguistique peuvent être source d'une valeur esthétique sui generis pour une œuvre littéraire.

\subsubsection{Sur les risques de l'usage des concepts nomades}

La circulation des savoirs et la reconfiguration des champs de recherche scientifique donnent lieu à l'émergence des concepts dits nomades. ${ }^{6}$ Il s'agit des concepts qui

\footnotetext{
${ }^{6}$ La notion de concept nomade (travelling concept) a été forgée par Bal [5].
} 
circulent par la voie de l'emprunt entre les disciplines scientifiques, les périodes historiques et les communautés académiques. Ce phénomène est bien connu dans tous les domaines. Si l'emprunt d'une notion comporte indéniablement un potentiel heuristique qui peut s'avérer bénéfique en termes de réflexion, il n'en reste pas moins qu'il produit des effets majeurs en ce qui concerne son contenu et les conditions de sa validité et peut, de fait, conduire à sa distorsion. On peut l'illustrer à l'exemple de la notion de traduction qui est elle-même un concept nomade dont la signification change selon la discipline qui l'emprunte. La théorie de la traduction emprunte également des notions à d'autres disciplines scientifiques, comme l'illustre, par exemple, la notion d'équivalence. Il s'agit d'une notion phare de la théorie linguistique de la traduction qui a été empruntée à la logique [42, p. 80]. Elle illustre également très bien les risques liés à l'emprunt des concepts que nous venons de mentionner. En effet, la reprise de la notion d'équivalence en théorie de la traduction a conduit à modifier aussi bien son contenu que ses conditions de validité. S'agissant du contenu, l'équivalence ne désigne plus, comme en logique, une relation univoque réversible, c'est-à-dire une relation bidirectionnelle entre deux éléments, mais une relation d'identité. De fait, le caractère bidirectionnel de la notion logique s'en trouve totalement éludé. S'agissant des conditions de validité de l'équivalence, elles ont également été profondément modifiées. Si en logique, la validité de l'équivalence est liée aux valeurs de vérité, en théorie de la traduction elle est liée à l'existence d'une entité de comparaison (tertium comparationis) en dehors de la langue et indépendante de toute langue. Faute de redéfinir une notion pour l'adapter à son nouveau domaine d'application, l'emprunt conduit à la transposer naïvement au mépris du fait que sa validité se limite à un domaine précis [39, p. 207]. C'est ainsi qu'une notion devient inapte à satisfaire le postulat méthodologique d'adéquation selon lequel une notion - pour être adéquate - doit être définie de manière à être compréhensible non seulement pour son auteur, mais aussi pour les autres [39, p. 226]. Par conséquent, si l'emprunt des notions et de théories, et donc l'emploi des notions nomades comporte indéniablement un potentiel en termes de l'évolution des connaissances et d'innovation, il n'en reste pas moins qu'il exige de la prudence et nécessite un examen critique de ses conséquences. Cet examen vise à déterminer en premier lieu la signification du concept qui lui est conférée par son auteur et, en second lieu, à déterminer les modifications de la signification du concept dues à sa transposition dans un contexte différent. En troisième lieu, il s'agit de déterminer, dans quelle mesure les concepts importés s'intègrent non seulement dans la théorie du nouveau champ, mais aussi dans sa pratique. C'est justement à ce type d'examen critique que nous souhaitons soumettre la théorie de Bhabha dans le présent article. Pour le réaliser, les instruments de la critique sceptique tels que le principe d'isosthénie et les tropes sceptiques peuvent être mobilisés. Les tropes sceptiques sont des arguments qui permettant d'identifier les erreurs de raisonnement tels que la contradiction, le cercle vicieux, la régression à l'infini ou l'acceptation sans preuve. Le principe d'isosthénie permet de considérer qu'à toute théorie scientifique une théorie de valeur équivalente peut être opposée. C'est la raison pour laquelle toute théorie mérite un examen minutieux, libre de préjugés qui permet de déterminer si elle peut être opérationnalisée dans un autre domaine et, si tel n'est pas le cas, de préciser les raisons qui conduisent à la rejeter. S'agissant 
des concepts nomades, la théorie de Bhabha, en utilise au moins deux, à savoir la traduction et l'hybridité. L'examen de ces notions dans les développements ci-après montrera qu'en adoptant ces concepts, il en modifie sensiblement la teneur, les limites, voire la valeur axiologique.

\subsubsection{Sur l'usage des métaphores conceptuelles}

S'agissant des métaphores conceptuelles, Bhabha en fait un usage prolifique. À titre d'exemple on peut citer le tiers-espace, « l'au-delà », l'espace « interstitiel » [7, pp. 31-32] ou l'espace « entre-deux » [7, p. 94], « la traduction culturelle » [7, p. 394] ou encore « la cage d'escaliers » [7, p. 36] et le « dé-placement » [46, p. 98]. Ce qui frappe dans l'usage de ces métaphores c'est le flou des concepts qu'elles sont censées désigner. L'origine de ce flou théorique peut résider dans la difficulté définitoire ou dans l'utilisation de la notion. En règle générale, le flou résulte de l'absence de définition et du caractère diffus de la signification conférée à ces concepts. Sans être critiquable en soi, le langage métaphorique prête néanmoins le flanc à la critique lorsque la métaphore se substitue à la définition et vise à faire l'économie de l'effort définitoire et de la justification. Si ce flou théorique n'est pas immédiatement identifiable pour un lecteur peu averti compte tenu du style dense de l'écriture, de la syntaxe complexe et de l'imbrication des lignes d'argumentation, il devient observable à mesure que le lecteur s'en familiarise. L'utilisation des métaphores conceptuelles ne lève pas l'écran des significations imprécises ou obscures, mais au contraire, amplifie le flou. Certains auteurs estiment toutefois, que l'absence de définitions et le déficit de clarté qui en résulte sont voulus par Bhabha [46, 54]. Comme d'ores et déjà annoncé, nous nous concentrons ci-après sur trois éléments clé de sa théorie et examinerons successivement les notions de traduction culturelle, d'hybridité et de tiers-espace. Outre l'ouvrage de Bhabha lui-même [7], notre exposé exploite les analyses réalisées par les chercheurs d'horizons différents [46, 53, 54].

\subsection{La traduction culturelle}

Dans la conception de Bhabha, la traduction ne s'entend pas au sens d'une opération linguistique. Il le dit d'ailleurs lui-même : «(...) j'utilise ce mot non au sens linguistique strict (...), mais comme motif ou trope, comme Benjamin suggère de le faire (...) » [8, p. 98]. Il va donc sans dire que ce n'est pas de traduction interlinguistique qu'il s'agit ici, mais de la traduction culturelle. En effet, il entend le processus de traduction non pas comme la transformation d'un signe linguistique en un autre et la resémantisation qu'emporte une telle transformation, mais comme un mécanisme culturel fondamental [46, p. 131], le modus operandi de l'hybridité. Ce n'est pas simplement un transfert, mais une véritable «trans-formation » et la valeur transformatrice de la traduction réside en une articulation novatrice - la traduction - des éléments en présence qui ne sont ni l'un ni l'autre, mais donnent lieu à l'émergence d'un objet nouveau. La transformation qui s'opère dans la traduction culturelle affecte, selon Bhabha, tous les éléments impliqués, l'original compris. C'est pourquoi la présomption d'un original stable permettant une traduction au sens d'une 
imitation est tout simplement intenable. Par conséquent, la notion même d'original s'en trouve remise en cause [46, p. 133]. La traduction en tant qu'opérateur de l'hybridité ne permet donc pas la synthèse des oppositions mais - au contraire - participe de la différenciation du sens résultant de l'hybridation. De fait, la traduction culturelle a pour effet d'accroître et non de réduire la complexité.

Dans cette conception singulière, les mécanismes de la traduction opèrent au-delà de la langue et correspondent à la déconstruction et la reconstruction des significations dans les zones de contacts hybrides aux limites des cultures. Il en résulte que pour Bhabha, la traduction est donc d'abord « une transformation de valeur » [7, p. 82] ${ }^{7}$ visant à permettre aux cultures de réviser leurs propres systèmes de références, leurs normes et leurs valeurs dans un processus d'aliénation sui-référentielle. C'est ce qui semble résulter de sa définition quelque peu cryptique de la traduction «Par traduction, je désigne avant tout un processus qui implique toujours, pour que le sens culturel soit objectivé, un processus d'aliénation et de secondarité par rapport à lui-même » [8, p. 98]. La traduction culturelle opère non seulement entre les cultures différentes, mais aussi au sein d'une même culture et produit des effets d'aliénation ce qui dynamise la culture en tant qu'entité stable et la rend instable. Par ailleurs, le processus de traduction constitue un lieu de lutte politique et culturelle $[46, \mathrm{p} .131]$ où s'opère en permanence un déplacement de la signification. Ce déplacement est constitutif de l'élément translationnel, la principale caractéristique de la culture, qui in fine s'avère n'être rien d'autre que l'hybridité permanente [46, p. 132].

En définitive, Bhabha donne à la traduction une définition large et aux contours flous, dans la mesure où il la conceptualise comme "une manière de comprendre le monde sans le réduire à une langue, mais de le comprendre à travers la traduction en donnant sa place à toute tradition culturelle et à tout texte culturel $»^{8}[1$, p. 173] Si une conception aussi large est spécifique de la traduction peut s'expliquer par la perspective singulière à partir de laquelle elle est articulée, à savoir à partir de la perspective des migrants, placés bon gré mal gré aux marges de la société d'accueil, il n'en reste pas moins qu'elle autorise toute sorte de lecture. Une telle conception large et floue de la traduction participe de la dilution du concept de la traduction.

\subsection{L'hybridité}

La notion d'hybridité, omniprésente dans l'œuvre de Bhabha, constitue un outil permettant de décrire de manière spécifique les contacts entre les cultures. C'est également la notion qui a donné lieu à une importante réception dans la littérature et à une riche controverse, notamment en raison de la diversité de ses définitions. L'une des définitions désigne l'hybridité comme « un processus permettant l'émergence de quelque chose de nouveau et méconnaissable, un nouveau champ de négociation

\footnotetext{
7 Pour décrire cette transformation, Bhabha utilise le terme anglais de transvaluation Struve [46].

${ }^{8}$ Notre traduction du passage original : "a way of understanding the world, not to reduce it to one language, but to understand the world by understanding translation, giving any particular cultural tradition or cultural text its own space”.
} 
de la signification et de la représentation » [46, p. 97]. Ce concept doit permettre de repenser les cultures à partir des positions intermédiaires et d'appréhender les phénomènes situés au-delà du familier. S'agissant de l'origine de la notion, il y a lieu d'observer qu'elle n'a pas été forgée par Bhabha, mais procède de deux sources distinctes : biologique et philologique. En biologie, elle désigne le croisement des espèces. Transposée à l'anthropologie raciale ${ }^{9}$ au $\mathrm{XIX}^{\text {ème }}$ et $\mathrm{XX}^{\text {ème }}$ siècle, elle a acquis une connotation éminemment péjorative qui est toujours opérante. En philologie, elle a été problématisée notamment par Mikhail Bakhtin [4] dans ses travaux sur le dialogisme et la polyphonie dans le discours. Si l'on conçoit le contact culturel en termes de diffusion des cultures, l'hybridité constitue à la fois le lieu et le résultat de ce contact [46, p. 100]. Dans cette acception, l'hybridité ne permet pas l'annulation des différences culturelles et ne conduit donc pas à la synthèse de deux cultures opposées. Au contraire, elle produit une sorte de mélange hétérogène de deux positions antagonistes. Ce mélange a toutefois ceci de particulier qu'il permet l'émergence de « quelque chose de différent, de neuf que l'on ne peut reconnaître » [8, pp. 99-100]. L'hybridité résulte d'un processus de différenciation. Il consiste à déconstruire en permanence les dichotomies et à reconstruire les identités culturelles, tant individuelles que collectives. Il en résulte que la notion d'hybridité décrit un processus à la fois de déconstruction et de reconstruction dont le résultat consiste en un objet nouveau hétérogène et méconnaissable [46, p. 102]. Cet objet nouveau est méconnaissable en ce sens qu'il «n'est ni l'un ni l'autre » [7, p. 71] et, en tant que le résultat de la déconstruction, il ne peut être rattaché à un quelconque original. Les processus d'hybridité se déroulent à des niveaux différents et revêtent des aspects différents. C'est pourquoi l'hybridité s'exprime au sein des espaces différents, à savoir [46, p. 100] :

(a) Un espace de mixité dans lequel se produit un mélange culturel au-delà de la synthèse des oppositions dialectiques au sens hégélien permettant l'annulation des différences;

(b) Un espace discursif dans lequel s'opère la transformation des symboles en signes. Dans cet espace, la signification ne peut plus être appréhendée et l'univocité disparaît pour faire place aux phénomènes du déplacement du sens. Le mécanisme à la base de ce phénomène consiste à réinterpréter un symbole univoque pour le fixer en un signe de différence culturelle. Dès lors, l'hybridité désigne « le déplacement de valeur du symbole au signe qui fait se cliver le discours dominant (...) » [7, p. 212]. Le symbole ainsi transformé devient ambigu, vague, voire illisible [46, p. 114].

(c) Un espace d'action dans lequel survient la crise de l'autorité coloniale, et enfin,

(d) Un espace d' ambivalence affective.

En outre, l'hybridité peut prendre plusieurs formes, à savoir : le déplacement, la dissémination et la réitération [46, p. 114]. Enfin, sur le plan de l'identité

\footnotetext{
${ }_{9}$ Voir, p. ex. Arthur de Gobineau Essai sur l'inégalité des races humaines. Paris, Firmin Didot, 1853.
} 
subjective, l'hybridité entendue au sens psycho-analytique correspond à « l'identification d'un sujet à travers un autre objet, un objet d'altérité » [46, p. 117]. L'hybridité fonctionne comme « une transition entre les espaces définis par les identifications fixes et ouvre une place pour la différence (...) » [46, p. 117]. Elle opère dans un espace qui lui est propre, désigné comme « le tiersespace », et qui s'identifie à elle. Bhabha le précise expressément : " l'hybridité est (...) le 'tiers-espace' qui rend possible l'émergence d'autres positions » [8, p. 99]. Il en résulte que les notions d'hybridité et de tiers-espace s'identifient l'une à l'autre et les termes qui les désignent sont des synonymes.

Avant de poursuivre, il semble utile de s'interroger sur les aspects de la notion d'hybridité susceptibles de revêtir un intérêt en termes de traduction interlinguale. Sur le plan linguistique, la notion d'hybridité renvoie aux phénomènes de créolisation et de métissage dans un contexte plurilingue. Sur le plan de la traduction interlinguale, elle renvoie aux notions telles que la communication interculturelle [26], la transculturalité, la transtextualisation [43, p. 49] et la transdifférence. À notre avis, plusieurs éléments ne manquent pas d'attractivité en ce qui concerne la traduction, à savoir :

(a) L'idée de l'émergence d'un objet nouveau et hétérogène. Cette idée est intéressante, car on peut considérer, en effet, que le texte issu du processus de la traduction correspond à un tel objet hybride, dans la mesure où il présente les éléments de la langue cible et de la langue source. Ce mélange hybride peut se manifester, par exemple, à un stade précoce de la traduction lorsque le travail terminologique et stylistique n'est pas encore achevé. Mais il peut aussi résulter du littéralisme retenu comme option méthodologique ou d'une volonté de souligner l'étrangeté (Fremdheit) du texte original.

(b) L'idée que l'objet nouveau, issu du processus d'hybridité, ne peut plus être rattaché à un original, est séduisante pour le traducteur, dans la mesure où elle modifie fondamentalement le statut de celui-ci et ouvre au traducteur un espace de liberté et de créativité que la conception classique de la traduction lui refuse.

(c) L'idée selon laquelle cet objet est construit par un individu à partir de sa position d'intermédiaire, déterminée par le fait de se situer dans un espace de « l'entre-deux ». Elle est intéressante, dans la mesure où elle thématise la position du traducteur en tant que médiateur entre les différents univers sémantiques et culturels.

Nonobstant leur attractivité, ces éléments suscitent des interrogations, notamment quant à leur applicabilité concrète à la traduction interlinguale, notamment juridique. Nous y reviendrons dans la section consacrée à la critique des notions de Bhabha. 


\subsection{Le tiers-espace}

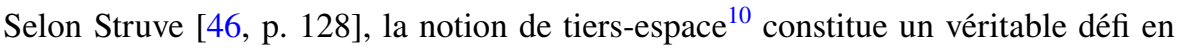
raison de sa polysémie. En effet, elle renvoie à :

(a) Une notion théorique qui se soustrait à toute définition,

(b) L'expression esthétique et éphémère de la différence culturelle,

(c) Le lieu d'affrontement (Kampfplatz) culturel et politique qui se crée au sein même du discours de l'autorité coloniale. Pour autant que nous avons compris, l'hybridité qui opère dans l'espace de transition qu'est le tiers-espace fait imploser ce discours et avec lui les systèmes de valeurs, les limites et les frontières qui lui sont inhérents.

La métaphore conceptuelle de tiers-espace articule la dimension spatiale de la notion abstraite d'hybridité [46, p. 121]. Elle constitue un élément de la sémantique de l'espace développée par Bhabha en combinaison avec d'autre mots tels que « l'entre-deux », « l'au-delà », « l'espace interstitiel » ou « l'interstice » [7, pp. 31-32]. Le tiers-espace est le lieu de l'hybridité [7, p. 94], d'une part, et d'autre part, un espace de traduction [7, p. 71]. Dans cette sémantique, le tiers-espace constitue l'articulation spatiale de l'hybridité comme :

(a) L'espace de représentation et l'espace discursif,

(b) L'espace textuel dans lequel les significations sont déconstruites et reconstruites dans le langage,

(c) L'espace de dé-placement de la valeur du symbole dans lequel un symbole univoque est transformé en signe polysémique qui matérialise la différence culturelle,

(d) L'espace de transition dans lequel s'exprime la différence culturelle [46, p. 123] et enfin

(e) L'espace de la zone de la différence culturelle, c'est-à-dire un espace de transition entre les binarités culturelles et linguistiques.

Le tiers-espace, éphémère et virtuel [46, p. 124], « porte le poids de la signification » [7, p. 94] et matérialise la zone de la différence culturelle dans laquelle s'opère l'hétérogénéisation des binarités. Les processus d'hybridité à l'œuvre dans le tiersespace ne permettent pas le dépassement des oppositions inhérentes à deux cultures distinctes au sens de la Aufhebung hégélienne qui permet d'annuler la dialectique des oppositions binaires. Au contraire, dans l'espace de la différence culturelle se crée un mélange hétérogène [46, p. 101] de sorte que les processus d'hybridité conduisent en toute logique à l'émergence d'un objet hybride. Dans cette espace

\footnotetext{
${ }^{10} \mathrm{Si}$ le terme de «tiers-espace » est une création de Bhabha, rappelons que la notion d'espace constitue un des piliers de la théorie de l'espace de Georg Simmel qui distingue différents types d'espaces : physique, social, symboliques et imaginaires Müller-Funk [30].
} 
éphémère de transition, l'hybridité se manifeste sur trois niveaux [46, pp. 123-124], à savoir :

(a) la processualité,

(b) la temporalité et la construction de l'histoire et

(c) la formation des communautés.

En ce qui concerne les deux derniers niveaux, les développements de Bhabha et de ses commentateurs sont complexes et éloignés de la réalité pratique de la traduction linguistique. C'est pourquoi, nous ne les discutons pas. En revanche, il en va autrement de la processualité. Elle joue un rôle majeur au regard de la notion de tiers-espace, dans la mesure où l'hybridité ne peut opérer qu'entre les entités instables impliquées dans une dynamique de l'interaction. Le tiers-espace en tant que zone de transition est le lieu où se déroulent en permanence les processus signifiants. C'est précisément là que le symbole figé dans son univocité disparaît au profit du signe polysémique. En d'autres termes, il est impossible de fixer le sens, ne serait-ce que temporairement. D'où l'absurdité de l'idée d'un original antérieur à la représentation [46, p. 124].

La notion de tiers-espace présente un certain intérêt du point de vue de la traduction interlinguale, car elle permet de problématiser la zone dans laquelle se construit le sens d'un texte dans une interaction réciproque et récursive qui a lieu en permanence entre les langues, les cultures, le(s) texte(s) et le traducteur. C'est donc un outil heuristique qui permet de décrire « la zone de contact » [33] au sens d'un espace d'interaction sociale. À notre avis toutefois, ces notions s'inscrivent davantage dans une sémiotique de la traduction [24] que dans la traduction dans son acception classique.

\section{3 Éléments de critique}

\subsection{La traduction}

Au vu de l'extension importante que Bhabha confère à la notion de traduction et de l'usage métaphorique ${ }^{11}$ qu'il en fait se pose la question de savoir en quoi la notion de traduction culturelle peut être utile à la traduction juridique comme pratique interlinguistique et pragmatique. De ce point de vue, plusieurs reproches sur le plan théorique et pratique peuvent être formulés à l'égard de la notion de traduction culturelle telle que Bhabha la conceptualise. Sur le plan théorique, les éléments qui prêtent le flanc à la critique sont les contours flous de la notion de traduction culturelle, la conception agonistique de la traduction et la reprise des lieux communs concernant la traduction interlinguistique. Sur le plan pratique, il semble nécessaire de revenir sur le sol raboteux de la pratique de la traduction. À cet égard, la critique majeure qui

11 Pour un exposé détaillé, voir Wagner [53]. 
peut être formulée concerne la difficulté d'articuler la notion de traduction culturelle à la praxis de la traduction juridique.

\section{(a) Sur le plan théorique}

La critique la plus importante concerne la nature diffuse de la notion de traduction culturelle et le flou qui entoure l'objet théorique visé par Bhabha résultant de l'extension qu'il confère à la notion de traduction. En raison de cette extension, et comme l'observent à juste titre certains auteurs, la notion de traduction culturelle « n'a rien avoir avec les langues et leur diversité, mais vise le transfert des représentations, des valeurs, des pratiques et des schèmes conceptuels » [53, p. 1]. Si nous souscrivons à cette opinion, il nous semble qu'elle mérite d'être nuancée. En effet, l'idée selon laquelle la traduction consiste en une transformation de valeur [7, p. 82] peut emporter l'adhésion du linguiste, dans la mesure où la traduction modifie effectivement la valeur d'un signe linguistique en le transposant dans un autre univers sémantique. En outre, la traduction interculturelle et la traduction interlinguale ont partie liée en raison de la nature discursive des représentations, des pratiques et des schèmes conceptuels. En d'autres termes, tous ces éléments se constituent non seulement dans le langage, mais surtout dans une langue particulière. La langue sert donc de médium de constitution des représentations et des valeurs, d'une part, et d'autre part, elle fournit en tant que système de signes le cadre de références commun spécifique à chaque culture, chaque langue et chaque société [37, p. 254]. Ce sont précisément les contours flous de la notion de traduction culturelle qui permettent cette lecture « linguistique ». Cependant, en l'absence de définition précise, le reproche du flou demeure, car la traduction culturelle en tant que l'objet théorique visé par Bhabha semble impossible à appréhender. Cette impossibilité se manifeste dans l'alternance constante entre l'acception classique du terme de traduction qui renvoie à la traduction interlinguale et son acception métaphorique qui renvoie au concept de traduction culturelle [53, p. 6]. La référence à Benjamin, ${ }^{12}$ convoquée pour servir de caution plutôt que de justification théorique de la traduction culturelle et de sa nature transformatrice ne convainc pas [53, p. 4]. En isolant les propos de Benjamin de leur contexte initial, Bhabha occulte, en effet, le fait que Benjamin parle des langues et non des cultures [53, p. 5]. Par ailleurs, en assimilant l'objet nouveau, issu des processus d'hybridation dans la traduction culturelle à ce que Benjamin désigne comme « le caractère étranger ${ }^{13}$ des langues » [7, p. 391], Bhabha passe sous silence le fait que pour Benjamin le caractère étranger des langues procède de la confusion babélienne et de la nature imparfaite des langues naturelles envisagées par rapport à une langue pure de la révélation et non de l'action transformatrice de l'hybridité, source de «l'étrangeté » en traduction culturelle. Le flou de l'argumentation de Bhabha quant à la justification de sa notion de traduction culturelle par la référence

\footnotetext{
${ }^{12}$ Le chapitre XI de son ouvrage Les lieux de la culture (2007) est précédé d'une citation extraite d'un texte de Benjamin de 1916 intitulé en allemand Über Sprache überhaupt und über die Sprache des Menschen.

13 Benjamin utilise le mot die Fremdheit.
} 
benjaminienne s'accompagne de la confusion entre l'usage littéral et l'usage métaphorique de la notion de traduction [53, p. 5]. On peut également relever une certaine confusion entre «le caractère étranger des langues » et « l'étrangeté ». Ces deux termes renvoient à la traduction des termes allemands das Fremde et die Fremdheit. S'agissant du caractère étranger des langues, il renvoie à la singularité de toute langue et à sa nature irréductible dans la diversité des autres langues. Chaque langue constitue, en effet, un univers sémantique autonome dont la grammaire n'implique pas des équivalents dans un autre univers sémantique. C'est en ce sens que « toute langue est étrangère »[47, p. 263] et les univers sémantiques fondamentalement asymétriques. Au moins depuis Humboldt, la vocation de la traduction consiste à rendre l'élément étranger (das Fremde), c'est-à-dire l'altérité et non l'étrangeté (die Fremdheit) [47, p. 277]. En traduction juridique, rendre l'élément étranger signifie rendre compréhensible son altérité et rendre audible la voix du justiciable étranger tout en évitant l'illusion d'isomorphie des cultures et des systèmes juridiques. En revanche, insister sur l'étrangeté d'un discours revient à nier la possibilité pour la traduction de rendre l'altérité de l'étranger en le faisant disparaître derrière l'écran de sa prétendue étrangeté. C'est pourquoi un excès de l'étrangeté « tue l'étranger » [10, p. 188]. Considéré du point de vue de la théorie de Bhabha c'est le contraire qui semble se produire : la traduction culturelle conduit à l'étrangeté de l'objet nouveau qui en est issu et non à la resémantisation de l'altérité dans la traduction de manière à la rendre accessible.

En outre, la conception éminemment agonistique de la traduction peut également faire l'objet de critique. En effet, s'il est possible d'analyser le droit en termes de lutte sémantique [15, 271], et la traduction juridique en termes de lutte pour le sens, il n'en reste pas moins que tant le droit que la traduction en tant qu'actes de communication visent à mettre fin à cette lutte en fixant le sens. La visée communicative impose donc de mettre un terme à la différenciation du sens. Or la conception agonistique de la traduction culturelle ne le permet pas.

Un autre reproche qui peut être formulé sur le plan théorique concerne la reprise par Bhabha des lieux communs concernant la traduction interlinguale. Ainsi, p. ex. il indique que «le transfert de signification ne peut jamais être complet entre différents systèmes de signification, ni même en eux (...) »[7, p. 292]. L'impossibilité de transfert linéaire de la signification et l'intraduisibilité résiduelle étant des phénomènes bien connus tant en théorie de la traduction [14, 41] qu'en sciences sociales [34, 36] et en droit [9, 59], l'affirmation de Bhabha s'assimile donc à une banalité. De la même façon, l'idée selon laquelle la traduction n'est pas une imitation, mais une transformation fait partie du canon de la théorie linguistique de la traduction. En revanche, le point sur lequel cette théorie et la théorie de Bhabha divergent concerne la nature de cette transformation. Si pour Bhabha elle s'opère dans l'hybridation et consiste à produire un objet caractérisé en termes de «ni l'un ni l'autre », pour les théoriciens de la traduction la transformation consiste en une transmission appropriante qui s'opère dans l'interprétation [47, p. 36]. 


\section{(b) Sur le plan pratique}

Sur le plan pratique, la critique majeure susceptible d'être formulée à l'égard de la notion de traduction culturelle concerne son applicabilité à la praxis de la traduction juridique. En effet, la question principale que posent la traduction et l'interprétation interlinguales est de savoir comment gérer la distance culturelle entre la langue cible et la langue source. En matière de traduction juridique, la difficulté posée par la distance culturelle est à l'origine de problèmes pratiques importants. Ils peuvent être illustrés à l'aide de deux exemples dont l'un concerne la traduction écrite et l'autre l'interprétation.

En ce qui concerne la traduction écrite, notre exemple de difficulté de la gestion de la distance culturelle concerne le terme allemand de Zugewinngemeinschaft. Il s’agit du régime matrimonial de droit commun en Allemagne ( $\$ 1363 \mathrm{du}$ Code civil allemand, BGB). En règle générale, ce terme est traduit en français par « communauté de biens réduite aux acquêts ». Le problème de cette traduction est qu'en réalité, le régime matrimonial allemand n'est pas une communauté de biens, mais une séparation de biens [22, p. 734]. Quelle serait donc la traduction culturelle adéquate au sens de Bhabha ? Dans la logique de production d'objets hybrides mettant l'accent sur l'étrangeté de l'étranger faudrait-il alors traduire " communauté de biens réduite aux acquêts s'analysant effectivement comme une séparation de biens » ? L'intelligibilité du texte traduit et l'efficacité de la communication en droit en pâtiraient de toute évidence. Il nous semble donc qu'une solution adéquate consisterait à conserver "la communauté de biens réduite aux acquêts » et d'assortir la traduction d'une explicitation, à condition que la situation concrète de la traduction le justifie. Pour éluder l'aspect de la mise en commun des biens des époux, le terme de «participation aux acquêts » pourrait éventuellement être utilisé, mais la pertinence de cette solution dépend de la situation concrète dans laquelle s'inscrit la traduction. En réalité, la notion de traduction culturelle est inapte à résoudre ce type de difficultés concrètes. En effet, la communication, pour réussir, nécessite une synchronisation de deux flux de conscience au moyen des signes, que ce soient les signes d'une langue ou d'une culture, afin de créer un consensus minimal entre les partenaires de communication. Or l'élaboration de ce consensus exige à minima une décision permettant de fixer le sens au moins provisoirement. Or la théorie de Bhabha ne le permet pas, car elle vise la différenciation du sens à l'infini.

En ce qui concerne l'interprétation dans le cadre judiciaire, la difficulté de gérer la distance culturelle peut être illustrée à l' aide de l'exemple suivant :

Lors d'une audience, le juge demande au justiciable étranger en français :

«Par quel moyen êtes-vous arrivés en France ? Par voie maritime ou en avion $? »$

Et au justiciable de lui répondre en anglais :

"If you want to cut a tree, you never climb up and start cutting up there, but you concentrate on the roots".

Soit littéralement en français : 
«Si vous voulez coupez un arbre, vous ne grimpez pas tout en haut pour commencer à couper les branches du haut, mais vous vous vous concentrez sur les racines ».

Que signifie cette réponse dans cette situation ? Et que doit faire l'interprète pour assurer la communication dans une situation où les connaissances et les positions sociales sont distribuées de manière asymétrique et où l'arrière-plan culturel n'est pas partagé par les partenaires de communication. Il en résulte une rupture épistémique entre le juge et le justiciable qui peut conduire à l'agacement chez l'un et au sentiment d'être désavantagé chez l'autre. Quelle attitude doit adopter l'interprète dans ces conditions sachant que par devant les tribunaux il lui est interdit d'expliciter. En effet, il n'est pas considéré comme étant « un expert en culture » d'une part, et d'autre part, l'interdiction lui est faite de prendre position. Pour autant, le simple transcodage, c'est-à-dire une traduction littérale est inefficace. Quelle serait la solution du point de vue de la traduction culturelle ? Il semble que la réponse à cette question à partir de la théorie de Bhabha soit loin d'être évidente.

\subsection{L'hybridité}

Peu de notions ont suscité dans la littérature autant d'adhésion et de critique que la notion d'hybridité ${ }^{14}$. Le présent article n'a pas pour objet l'exposé exhaustif de cette critique en sciences sociales, mais il est permis d'observer que différents éléments justifient cette critique, à savoir : l'absence de définition et le caractère vague du concept qui en résulte, le fait de conférer une signification positive à une notion à connotation péjorative [46, pp. 166-167], la connotation politique et la prétention universalisante inhérente au concept [46, p. 169] ainsi que son essentialisation et l'absence de référent concret [46, p. 171]. De manière générale, on notera les déficits suivants de la notion :

(a) L'absence de définition claire qui contribue à la dilution du concept;

(b) Le caractère diffus de la notion qui résulte de la confusion entre le processif et le résultatif. En effet, l'hybridité est envisagée comme un processus, d'une part, et d'autre part, comme le résultat de ce processus.

(c) L'identité définitoire entre hybridité et tiers-espace [8, p. 99].

La nature sémiotique de la traduction, l'intrication de la culture et du langage dans le monde de la vie quotidienne (Alltagswelt) conçu a priori comme un monde intersubjectif de la culture $\left[39\right.$, p. 187] ${ }^{15}$ et la circulation des savoirs et des concepts mentionnés ci-dessus expliquent les tentatives de transposer la notion d'hybridité en théorie de la traduction. Cependant, il se pose la question de l'opportunité et de

\footnotetext{
14 Pour un exposé détaillé de la réception critique des concepts de Bhabha dans l'aire germanophone, voir p. ex. Struve [46] avec d'autres sources. S'agissant de la critique en France, voir p. ex. Bayart [6].

15 Schütz montre que le monde de la culture est un univers intersubjectif des significations et des contextes de sens dans lequel le sens se constitue intersubjectivement, c'est-à-dire dans l'interaction avec autrui.
} 
la pertinence d'une telle transposition. Sans nier l'importance des aspects culturels et leur influence déterminante sur le processus de la traduction interlinguale [49], il nous semble néanmoins judicieux de formuler les observations suivantes quant aux différentes idées présentes dans la théorie de Bhabha et susceptibles d'un transfert dans le domaine de la traduction interlinguistique.

\section{(a) Quant à l'idée de l'émergence d'un élément totalement nouveau}

Selon Sherry Simon, un texte est hybride, dans la mesure où il résulte de l'interpénétration de deux systèmes linguistiques. En ce sens, tout texte traduit est un texte hybride. Pour cet auteur toutefois, la notion de texte hybride reste réservée aux textes qui conservent les marques de cette interpénétration, c'està-dire qu'ils reflètent « les 'effets de la traduction' tels que les dissonances, les interférences, un lexique disparate, un manque de cohésion, une syntaxe peu conventionnelle (...) [43, p. 50]. Si du point de vue de la traduction littéraire les textes hybrides ainsi définis peuvent être considérés comme dotés d'une valeur esthétique en tant qu'une expérimentation littéraire, du point de vue de la traduction pragmatique, on peut s'interroger sur l'intelligibilité ${ }^{16}$ de ces textes. En effet, la charge de la construction du sens étant déplacée du traducteur vers le lecteur allogène, il lui appartient de se débrouiller avec les incohérences, le lexique inadéquat, la syntaxe inhabituelle, etc. Le caractère hybride exacerbé d'un texte traduit peut le rendre inintelligible, soit parce que la forme linguistique est incompréhensible, soit parce que les référents culturels et historiques ne sont pas identifiés. En définitive, produire un texte hybride en traduction pragmatique revient, selon nous à mettre en œuvre la méthode de traduction connue depuis Luther comme exotisation [28] dans une perspective de littéralisme strict. Cet asservissement au signifiant du texte source aboutit à un calque lexical et syntaxique dans lequel le texte traduit est contaminé par les éléments de la langue source de manière à occulter son altérité par l'écran de l'étrangeté. Il est donc permis d'être sceptique quant à l'intelligibilité de ce type de textes.

\section{(b) Quant à l'idée de la modification du statut de l'original}

Le statut de l'original est une question complexe en traduction qui appelle des réponses différentes selon que l'on se situe sur le plan de la traduction littéraire ou de la traduction juridique. S'agissant de la traduction juridique, il est intéressant de relever qu'en droit, l'original ne renvoie pas à un texte original, mais à un texte « reconnu officiellement comme faisant foi de la vérité dans une discipline donnée » $[17$, p. 205]. Cette reconnaissance officielle est constitutive de l'autorité authentique conférée au texte par un organe habilité à en faire une interprétation authentique et explique son statut sacro-saint. La

$\overline{16}$ Sur les formes d'inintelligibilité en traduction, voir Durr [13]. 
notion d'autorité authentique du texte explique le fait qu'en droit international, l'original se trouve éludé par le biais de la notion de version authentique. Lorsqu'un texte normatif ou une décision de justice sont rédigés en plusieurs langues, les différentes versions linguistiques constituent des versions authentiques, c'est-à-dire quelles sont reconnues officiellement comme investies de la même autorité authentique. Elles sont donc réputées équivalentes, aucune des versions ne pouvant revendiquer le statut de l'original. Les termes utilisés dans les différentes versions linguistiques sont présumés avoir le même sens. Bien entendu, il s'agit d'une fiction juridique qui permet de passer outre les différences linguistiques pour préserver l'unicité d'une disposition normative ou d'une décision de justice et se concentrer sur l'interprétation téléologique plutôt que de s'égarer dans les méandres de l'interprétation grammaticale. Toutefois, dans la pratique quotidienne de la traduction juridique qui correspond à la traduction « de langue à langue » et de « de droit à droit », il est impossible de faire abstraction de la notion de texte original en raison de la force probante qui lui est attachée. C'est pourquoi, et contrairement à l'affirmation selon laquelle « (...) la traduction étant une imitation, l'original peut être copié, modifié, transformé en simulacre, etc. » [8, p. 99], en traduction juridique ces altérations de l'original ne sont pas concevables.

\section{(c) Quant à l'idée du traducteur comme agent de la médiation}

Concernant cette idée, on observera qu'elle ne représente rien de nouveau sous le soleil de la théorie de la traduction, car elle a été thématisée à de nombreuses reprises dans la littérature, notamment dans le contexte de la médiation $[26,58]$. En tant que pratique communicative, la traduction peut s'analyser en termes de médiation interlinguistique, interculturelle et langagière [25, p. 633]. Dans l'interaction entre deux langues et deux univers culturels, le traducteur en tant que médiateur occupe la position du « méson », c'est-à-dire du moyen terme qui fait le lien entre deux autres termes. S'il est indispensable pour la mise en relation, il a vocation à disparaître dès que sa mission est accomplie [13, p. 329]. La position du traducteur en tant que médiateur est donc à la fois centrale et précaire.

\subsection{Le tiers-espace}

Comme la notion d'hybridité, celle de tiers-espace a fait l'objet de critiques de la part des spécialistes des sciences de la culture ${ }^{17}$ qui ont notamment souligné son caractère extrêmement abstrait [46, p. 173]. S'il ne nous appartient pas d'entrer dans le détail de cette critique dans le cadre du présent article, il est néanmoins possible de relever des éléments qui, à notre avis, prêtent le flanc à la critique. Du point de vue de la traduction pragmatique, trois reproches peuvent être formulés.

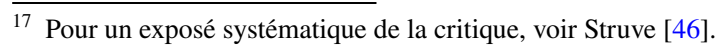


(a) Le premier reproche concerne l'identité des notions d'hybridité et de tiersespace, affirmée par Bhabha lui-même. Elle conduit à la circularité des concepts et à la synonymie des termes [46, p. 117]. Ces notions se déterminant réciproquement, le reproche de diallèle ${ }^{18}$ peut être formulé sur le plan logique. Ces déficits matérialisent l'absence de l'effort définitoire et participent de la dilution des concepts. Or il est notoire que l'intelligibilité d'un concept est inversement proportionnelle au nombre de ses extensions. En d'autres termes, plus un concept a d'extensions, moins il est intelligible.

(b) Le second reproche a trait à l'absence de possibilité d'intégration de l'altérité. Elle résulte de l'exacerbation du caractère hétérogène de l'objet nouveau issu des processus d'hybridation à l'œuvre dans le tiers-espace d'une part, et d'autre part, de l'impossibilité de synthèse des oppositions au sens de Aufhebung hégélienne. [46, p. 101] Les textes hybrides mentionnés précédemment, contaminés par des éléments lexicaux, syntaxiques et stylistiques de la langue source, illustrent bien cette exacerbation de l'hétérogène. Or la traduction pragmatique vise à produire des textes qui ont du sens [16, p. 51]. Cette visée implique donc la nécessité de fixer au moins provisoirement le sens d'un mot, d'une proposition ou d'un texte. Or fixer le sens signifie de transformer un signe polysémique en un signe univoque. À la différence de la traduction culturelle, qui fait imploser un symbole univoque en des signes polysémiques, la traduction interlinguistique met un œuvre un processus différent. En effet, la production du sens implique, selon nous, un processus de resémantisation dans lequel le signe de la langue source initialement univoque est d'abord redéployé dans toute sa polysémie pour se voir conférer ensuite un sens dans la langue cible. Ce processus implique pour le traducteur d'être en mesure de dépasser les oppositions qui résultent de la polarité du sens [37, p. 272] et de l'oscillation de la signification entre le sens objectif, occasionnel et subjectif d'un signe linguistique [37, p. 118]. Ce dépassement s'opère précisément dans le processus de la synthèse hégélienne des oppositions dialectiques (Aufhebung) ${ }^{19}$ avec ses trois étapes de negare, conservare et elevare. Ces étapes peuvent s'analyser comme suit au regard de la traduction linguistique : la négation (negare) correspond à l'annulation de l'univocité, la conservation (conservare) correspond à l'intégration de la différence et, enfin, « l'élévation » (elevare) correspond au dépassement de la polysémie et à la fixation du sens dans la langue cible grâce à la sélection des unités lexicales et syntaxiques et des moyens stylistiques suivant le principe de la pertinence pour produire un objet nouveau sous forme de texte traduit. Il va sans dire qu' en traduction interlinguistique, cette synthèse est fréquemment partielle et graduelle en raison de l'asymétrie des univers sémantiques et des systèmes juridiques.

\footnotetext{
18 Le diallèle, du grec diallèlos, désigne ce que l'on appelle aussi « cercle vicieux » ou « inférence réciproque », et qui consiste à définir un terme ou à démontrer une proposition au moyen d'un autre terme ou d'une autre proposition, qui ne peuvent eux-mêmes être définis ou démontrés que par les premiers (cité suivant l'Encyclopédie Universalis en ligne : https://www.universalis.fr/encyclopedie/diallele/).

19 Sur la notion hégélienne de Aufhebung, voir Vieweg [51].
} 
(c) Le troisième reproche concerne la sémiose à l'infini dans la zone de transition délimitée par le tiers-espace ce qui conduit à la différenciation du sens dans une progression à l'infini et, donc, à l'impossibilité de le fixer. Du point de vue de la traduction pragmatique, il s'agit d'une objection majeure. En effet, si l'on peut accepter l'idée selon laquelle la communication intersubjective met en permanence en œuvre des processus signifiants, l'impossibilité de fixer le sens d'un texte nous semble inacceptable, dans la mesure où la traduction a précisément pour vocation de mettre un terme au moins provisoirement à la sémiose. C'est pourquoi la traduction ne peut se passer de la décision du sens. Certes, avant de prendre cette décision, le traducteur est effectivement confronté à la polysémie des signes et l'hétérogénéité des référents culturels, historiques, etc. ce qui l'oblige à faire le choix entre les différentes options de traduction. À défaut, le risque de l'éclatement du texte à traduire en un ensemble des signes polysémiques, dépourvus de sens, fait échouer toute entreprise de traduction interlinguistique.

En définitive, la théorie de Bhabha conceptualise une situation de communication spécifique, envisagée à partir de la perspective d'un migrant, c'est-à-dire d'un individu situé à la limite du groupe d'accueil. La différenciation du sens décrite dans les processus d'hybridité résulte de la différence de perspectives du migrant et du groupe d'accueil. Cette différence des perspectives est impossible à surmonter, car le postulat de la réciprocité des perspectives est inopérant [39, p. 340]. Grâce à ce postulat, on peut considérer que l'échange de perspective avec mon partenaire de communication permet de prendre pour évident le même cadre des références. Il va sans dire qu'en réalité, il est impossible pour un individu d'échanger sa position et sa perspective avec un autre, dans la mesure où elles sont déterminées par l'inscription singulière de tout individu dans le monde de la vie. Cependant, postuler la possibilité d'adopter la perspective de l'autre permet de créer une base minimale de la communication. La réciprocité des perspectives étant inopérante dans les processus d'hybridité, ils ne peuvent conduire qu'à l'implosion du discours, l'impossibilité du dialogue et, in fine, à l'absence de communication.

$\mathrm{Au}$ vu de ce qui précède, il semble donc que l'importation en traduction juridique des notions forgées par Bhabha, au demeurant floues, ne soit pas évidente malgré leur attractivité potentielle. Par ailleurs, il n'est pas interdit de se poser la question de l'utilité d'un tel transfert, dans la mesure où d'autres outils méthodologiques permettent de problématiser les questions d'interaction et d'influence réciproque entre la langue / la culture source et cible dans le processus de la construction du sens en traduction interlinguistique. Dans la section ci-après, il est proposé d'aborder ces questions à partir des notions telles que l'intervalle [10], l'entre-deux [56], la frontière [29], et la pertinence [38]. L'objectif visé consiste à montrer que la traduction interlinguistique, pour réaliser sa visée communicative, est nécessairement un lieu d'intégration de l'altérité. 


\section{La traduction interlinguale comme lieu d'intégration de l'altérité}

À titre liminaire, il semble judicieux de rappeler que l'altérité (Fremdheit) peut être envisagée de différents points de vue. De manière générale, l'altérité constitue une catégorie générale d'interprétation du monde et s'oppose à ce titre à la familiarité, c'est-à-dire à ce que l'on connaît bien (Vertrautheit) [40, p. 73]. Elle est donc nécessaire pour définir ce qui est familier. Ne désignant pas une propriété essentielle, mais une relation sociale, l'altérité n'est pas réservée à la situation spécifique d'un migrant, mais constitue un phénomène omniprésent. Elle se caractérise par une sensibilité élevée à la situation dans laquelle s'inscrit un acte de communication. Par conséquent, ce qui est perçu comme étranger dépend de la situation. Vecteur de l'altérité, l'étranger (Fremder) se caractérise par son positionnement en marge d'un espace socioculturel qui est un espace symbolique et intersubjectif, c'est-à-dire relationnel. Toute modification de cet espace peut modifier la position d'un individu au sein de cet espace en le poussant en dehors de ses limites ou en le reléguant à sa marge de manière à le caractériser comme «étranger » [30, pp. 135-136]. Ainsi, tout un chacun peut devenir étranger, même au sein d'un espace $a$ priori familier. Toutefois, l'altérité n'exclut pas a priori la possibilité d'intégration. En effet, entendue comme une « expérience différente du monde de la vie », l'altérité est un processus de transformation dans la durée qui ouvre la voie à l'intégration [40, p. 22]. Compte tenu du caractère ambigu de toute communication qui résulte de l'incertitude de la référence à laquelle renvoie un signe, l'altérité se définit également comme une nonidentité [44, p. 135] et implique, à ce titre, la présence simultanée de sémantiques distinctes, voire antagonistes. En traduction interlinguistique, l'altérité pose la question majeure de la gestion de ces sémantiques contradictoires, d'une part, et d'autre part, de la possibilité de leur intégration. Si la question de la coprésence des sémantiques contradictoires renvoie au phénomène d'hybridité et à l'espace interstitiel entre les langues et les cultures, la question d'intégration renvoie à la problématique de l'ouverture de la clôture opératoire entre les univers sémiotiques et le mécanisme de la production du sens.

\subsection{La transdifférence ou la gestion de l'hybridité en traduction interlinguale}

L'attractivité de la notion d'hybridité pour la traduction interlinguale s'explique sans doute par une certaine similarité entre les processus d'hybridation dans le tiers-espace et les processus à l'œuvre dans la traduction. En effet, si l'on considère l'hybridité comme un processus simultané de déconstruction et de reconstruction dont le résultat consiste à faire émerger un objet nouveau et hétérogène [46, p. 102], cette description résume assez bien ce qui se passe en traduction. La différence entre la notion d'hybridité de Bhabha et les processus d'hybridation à l'œuvre dans la traduction interlinguistique réside, à notre sens, dans la nature de l'objet nouveau qui en est issu. Pour Bhabha, cet objet est non seulement nouveau et hétérogène, mais aussi méconnaissable. Dans les processus de l'hybridité qui se déroulent dans le tiers-espace, les sémantiques antagonistes du migrant et du groupe d'accueil s'excluent mutuellement. Cette exclusion conduit à la différenciation du 
sens à l'infini qui produit un objet méconnaissable, dans la mesure où ni le migrant ni le groupe d'accueil ne peuvent s'y reconnaître. C'est pourquoi il peut être caractérisé comme étant «ni l'un ni l'autre » [7, p. 71]. Dans la traduction interlinguistique, la visée communicative de la traduction impose à rechercher une intégration des sémantiques contradictoires afin de rendre accessible la spécificité de l'étranger. Cette intégration peut être subsumée à la notion de transdifférence, définie comme « les processus de la constitution de sens où deux ou plusieurs univers de sens sont mis en relation sans pour autant niveler leur altérité ou leur différence réciproque » [44, p. 129]. La transdifférence, qui fait écho à la notion de sens défini par Luhmann comme « l'acquisition de signifiance dans la différence » [48, p. 171], décrit la simultanéité des sémantiques distinctes et souligne le caractère provisoire, instable et inhomogène de la constitution du sens [44, p. 129]. Elle constitue une caractéristique fondamentale de la communication, et donc aussi de la traduction en tant qu'acte de communication. L'intégration de la différence dans le texte traduit entraine des conséquences en ce qui concerne la nature du texte traduit considéré comme un objet nouveau, issu du processus de la traduction interlinguistique. En effet, s'il peut être qualifié d'hétérogène, car il porte les traces de la transdifférence, il ne saurait être méconnaissable au sens défini ci-dessus. Au contraire, issu d'un processus d'intégration de sémantiques distinctes, il peut être caractérisé comme « l'un et l'autre ». L'intégration des univers sémantiques hétérogènes visée dans la traduction opère dans l'idéal sur trois plans différents : la forme de la pensée (la cognition), la forme de l'action (la praxis) et la forme linguistique de manière à reconstruire le contexte de sens qu'elles créent [44, p. 142]. Cette reconstruction implique une pratique herméneutique qui intègre la pensée, l'action et la langue sans gommer, mais aussi sans exacerber les spécificité du texte rédigé dans une langue étrangère. La mise en relation des univers sémantiques distincts peut recourir à différentes stratégies. Ainsi, par exemple, lorsqu'une notion ne possède pas dans la langue cible la même extension que dans la langue source, une explicitation comprenant la traduction du contexte pragmatique peut être utile. Une autre stratégie pour gérer la co-présence de sémantiques distinctes peut consister à souligner la spécificité des éléments étrangers par une description détaillée tout en cherchant à atténuer l'effet qu'ils peuvent produire par le choix d'une interprétation permettant de les subsumer à un contexte plus familier [44, p. 171]. Pour reprendre l'exemple de la Zugewinngemeinschaft cité précédemment, on pourrait proposer une traduction qui décrit précisément ce régime pour finalement l'intégrer dans le schéma conceptuel bien connu de la séparation de biens. Compte tenu de ce qui précède, la notion de transdifférence est apte à rendre compte des mécanismes complexes permettant de gérer la présence simultanée de sémantiques différentes en traduction interlinguistique, la gestion de cette simultanéité étant un des défis majeurs en traduction. La transdifférence ne s'identifiant pas à l'intervalle entre les langues et les cultures créé dans le processus de la traduction, cette notion présente en outre l'avantage de ne pas être circulaire et de ne pas aboutir au diallèle. 


\subsection{L'espace interstitiel et le dépassement de la frontière entre les univers sémantiques}

La sémantique de l'espace comporte indéniablement un potentiel heuristique au regard de la traduction dans son acception classique. En effet, dans l'oscillation entre les entités langue /culture source et cible qui se produit au cours du processus de la traduction un espace vient à se créer. C'est ce qui explique sans doute l'attractivité de la notion de tiers-espace de Bhabha pour la théorie de la traduction. Si l'espace d'oscillation ouvert dans la traduction interlinguistique se rapproche du tiers-espace, il s'en distingue aussi. Il s'en rapproche par son caractère éphémère et symbolique. Il est éphémère, car il n'existe que pendant le processus de la traduction (energeia) ${ }^{20}$ et disparaît dès que ce processus cesse pour s'incarner dans la traduction en tant que texte concret (ergon). C'est un espace symbolique, car il se forme entre les langues et les cultures entendues comme systèmes de symboles. C'est également un espace instable dont les contours se modifient en permanence au gré des options de traduction qui s'offrent au traducteur. L'espace d'oscillation créé dans la traduction se distingue du tiers-espace par le fait que le choix par le traducteur d'une option de traduction fixe le sens et emporte, au moins provisoirement, la fin de la sémiose. L'espace d'oscillation entre deux systèmes linguistiques et culturels ainsi caractérisé peut être qualifié d'espace interstitiel. Il peut être appréhendé méthodologiquement comme un intervalle [10] ou comme un «entre-deux » [56].

La notion d'intervalle est intéressante, car elle permet d'articuler deux aspects de la traduction : la création de l'espace interstitiel, d'une part, et d'autre part, la position du traducteur en tant qu'intermédiaire. Conçu en termes d'intervalle, l'espace interstitiel, caractérisé ci-dessus comme un espace d'oscillation, correspond à la zone complexe d'interactions et d'interférences [10, p. 229] entre deux langues et deux cultures, créatrices « des mondes différents » [10, p. 49]. Dans cet espace interstitiel décrit par l'intervalle, le traducteur occupe une position paradoxale, à la fois intermédiaire et centrale. Sa position est d'abord celle d'un intermédiaire qui établit le lien entre deux espaces linguistiques et culturels distincts. S'il est indispensable à l'établissement d'une relation entre ces espaces, il est voué à disparaître dès qu'elle est établie. La nature provisoire du rôle dévolu au traducteur en tant qu'intermédiaire fonde la précarité de sa position et le postulat de sa nécessaire invisibilité. Mais la position occupée par le traducteur dans le processus de traduction se caractérise aussi par sa centralité. En effet, le traducteur en tant qu'agent se situe au centre de l'espace interstitiel et au centre du processus de resémantisation qui s'effectue par son truchement. Bien entendu, son inscription singulière dans le monde de la vie détermine les schémas culturels dans lesquels il a été lui-même socialisé tandis que l'ensemble des connaissances qu'il a acquises en tant qu'individu déterminent son aptitude à appréhender la spécificité des schémas culturels qui sous-tendent la langue source pour la restituer autant que faire se peut dans la langue cible. Le schéma culturel se définit comme «l'ensemble de valeurs, d'institutions et de systèmes qui caractérisent

\footnotetext{
${ }^{20}$ C'est la distinction classique de Humboldt thématisée dans l'Introduction au kavi. Elle permet d'appréhender la double nature de la traduction comme un processus et son résultat.
} 
un groupe social et permettant de s'orienter, à savoir les usages, les mœurs, les lois, les traditions, etc. » [40, p. 60]. C'est donc tous ces éléments, et non seulement le lexique et les structures grammaticales, que le traducteur a vocation à resémantiser autant que possible dans la langue cible. Le schéma culturel tel que défini ci-dessus sert également de schéma d'interprétation et d'expression. C'est un schéma standardisé, transmis par la tradition qui détermine la manière adéquate d'agir ou de se comporter dans une situation donnée $\left[40\right.$, p. 63] ${ }^{21}$. À ce titre, il permet de s'orienter dans un espace socioculturel. Le traducteur est le pivot et la pierre angulaire de la resémantisation en langue cible des schémas culturels étrangers (fremd) ou peu familiers (unvertraut) qui sous-tendent le texte source afin de permettre au destinataire de la traduction de s'y orienter grâce à une contextualisation adéquate tant au niveau local (unités lexicales et syntaxiques) que global (le texte). Ce travail de resémantisation s'effectue dans l'espace interstitiel décrit ci-dessus. Il consiste à subsumer les schémas d'interprétation d'un système linguistique et culturel à un schéma d'interprétation d'un autre système linguistique et culturel, à l'intégrer dans un contexte de sens concret afin de l'utiliser comme un schéma d'expression. C'est en cela que consiste la maîtrise d'un système de signes, et donc aussi, d'une langue [37, p. 254]. Cette opération de resémantisation constitue le cœur même de toute traduction. Elle participe de la compréhension et, donc, de l'intelligibilité du texte pour le lecteur. Nous pensons qu'il appartient au traducteur d'effectuer le difficile travail de resémantisation et de ne pas se contenter d'une traduction littérale qui a pour effet de transférer toute la charge d'établir la compréhension du texte à son destinataire. Concrètement, dans une traduction qui s'inscrit dans un contexte pragmatique et des langues de spécialité, la resémantisation s'opère sur plusieurs plans différents tels que la lexie, la grammaire, le registre de la langue et la prosodie sémantique, Pour le traducteur, il s'agit d'utiliser les schémas lexico-grammaticaux, c'est-à-dire les éléments d'une langue de spécialité [19], constitutifs d'un schéma d'expression [37, p. 253] sui generis. Cela conduit, le cas échéant, à s'éloigner de manière significative du texte source, voire à écrire un texte différent. Dans la resémantisation se révèle la nature doublement créatrice de la traduction : elle est créatrice de sens permettant « l'accord dans les jugements » [57, p. 135], d'une part, et d'autre part, elle crée un espace partagé de communication [36, p. 151]. L'opération de resémantisation sur le plan lexical, syntaxique, du registre et de la prosodie sémantique oblige le traducteur à opérer une sélection parmi toutes les options possibles en termes de solutions de traduction sur le plan terminologique, syntaxique et stylistique. L'espace interstitiel créé au cours du processus de traduction est le lieu de cette sélection. Elle joue un rôle capital en matière de traduction, car elle permet au traducteur de fixer le sens et, donc, de mettre provisoirement un terme à la sémiose. Or la sélection d'un élément signifiant parmi un ensemble d'autres éléments signifiants est constitutive du mécanisme de la pertinence $^{22}[38$, p. 51] qui préside à la constitution du sens [40, p. 11]. Nous reviendrons

\footnotetext{
21 Schütz utilise les termes de Auslegungsschema et Ausdrucksschema.

22 S'agissant de cette notion, il convient d'observer que nous n'opérons pas avec la pertinence d'extraction mentaliste conceptualisée par Sperber et Wilson, mais avec la notion de pertinence issue de la sociologie compréhensive conceptualisée par Schütz [37, 38] Cette notion a été opérationnalisée spécialement pour la traduction juridique par Durr [13].
} 
sur cette notion dans la section consacrée à la possibilité d'ouverture de la frontière entre les univers sémantiques distincts.

Une autre conception intéressante permettant d'appréhender l'espace interstitiel entre les langues et les cultures a été proposée par le philosophe Heinz Wismann [56]. Il nomme cet espace comme un « entre-deux » et le caractérise comme un milieu de réflexivité [56, p. 38] et comme un espace de récréation [56, p. 103]. Le milieu de réflexivité correspond à l'intervalle entre deux altérités et s'ouvre à quiconque se trouve en position d'intermédiaire. Celui-ci n'a pas tant vocation à transmettre des contenus par-delà les frontières des univers linguistiques, mais à réfléchir sur la manière d'exprimer ces contenus dans la langue d'arrivée. Cela suppose tout particulièrement une réflexion sur la grammaire et la syntaxe, puisque c'est à ce double niveau que se joue, selon Wismann, la différence culturelle entre les langues. Dans une communication, et donc aussi dans une traduction, il importe avant tout d'identifier cette différence pour explorer les possibilités grammaticales de l'exprimer non pas en soulignant son étrangeté qui peut produire un « effet de désarroi », mais au contraire mettant l'accent sur « son caractère étranger » et «l'effet de découverte » qui peut en résulter. Ce travail d'exploration des possibilités grammaticales de rendre compte de l'altérité et non de l'étrangeté d'un texte permet, en définitive, de ne pas se retrouver «lost in translation» [56, p. 14]. Cela infirme l'affirmation de Bhabha selon laquelle « dans l'acte de traduction, le contenu 'donné' devient étrange et étranger; et ceci (...) laisse le langage de la traduction, toujours confronté à son double, l'intraduisible - étranger et étrange » [7, p. 293]. Pour justifier cette affirmation, Bhabha convoque Benjamin à titre de caution en précisant que « le caractère étranger du langage est le noyau de l'intraduisible » [7, p. 292]. Il nous semble que cette affirmation procède d'une mécompréhension de la conception benjaminienne du langage. Pour Benjamin, en effet, la source de l'intraduisible ne doit pas être recherchée dans le « caractère étranger des langues », mais dans l'asymétrie des langues sur le plan syntaxique et lexical qui détermine non seulement ce qu'une langue permet d'exprimer, mais aussi la manière de l'exprimer. Cette asymétrie résulte des différences dans les modalités d'appropriation du monde et elle a pour effet de créer une tension « entre deux manières de se rapporter à la réalité (...), non pas sur le mode du reflet (...) mais sur le mode de la création de la réalité comme ce à quoi on est invité à se référer grâce à l'exploit linguistique » [56, p. 83]. Sur le plan lexical, l'asymétrie entre les langues peut s'expliquer par les « opérateurs culturels, c'est-à-dire les schèmes qui font que des univers de sens se coagulent autour des mots » [56, p. 78]. En réalité, ces opérateurs culturels correspondent à ce qui a été désigné comme « les franges de sens » (Sinnfransen), appelées aussi « horizons de sens », c'est-à-dire « les connotations ouvertes de sens attachées à un mot » [36, p. 241]. Sur le plan linguistique, ces « franges de sens» sont fixées et transmises sous forme de schémas d'interprétation [40, p. 69]. Elles font partie intégrante de la signification, mais restent intraduisibles [36, p. 279; 10, p. 24]. C'est pourquoi toute traduction concrète nécessite un choix, une décision de sens pour déterminer la manière adéquate de rendre compte, au moins partiellement, de ces horizons de sens. C'est également la raison pour laquelle toute traduction demeure imparfaite : 
« Traduire signifie toujours 'raboter' quelques-unes des conséquences que le terme original impliquait. L'interprétation, qui précède toute traduction, doit établir combien et lesquelles des conséquences illatives suggérées par le terme original peuvent être rabotées. Sans jamais être tout à fait sûr de ne pas avoir perdu un réverbère ultraviolet ou une allusion infrarouge » $[14, \mathrm{p}$. $110]$.

Si les horizons de sens relèvent de l'ordre de l'intraduisible ce n'est pas parce qu'ils sont «étranges », mais parce qu'étant perçus différements, ils font naître des associations différentes. C'est pourquoi, pour reprendre l'exemple de Benjamin cité par Bhabha, Brot en allemand n'est pas pain en français, car « ça se mange autrement, ça s'associe à d'autres situations que ce que le mot « pain » évoque dans le contexte culturel français » [56, p. 89]. Il en va de même de baguette en français et de butka paryska ${ }^{23}$ [pain parisien] en polonais. Si à l'origine, butka paryska était censée être une traduction de baguette, elle ne s'y identifie nullement malgré le fait d'être réalisée avec du blé, d'être une sorte de pain blanc et d'avoir la forme allongée. En effet, si butka paryska n'est pas baguette c'est parce qu'elle n'est pas perçue comme une baguette. A l'heure actuelle, on désigne en polonais baguette par bagietka qui est un emprunt du français. Mais bagietka ne veut pas toujours dire la même chose que baguette en l'absence d'associations identiques (camembert, béret) ou, à tout le moins, similaires. Pour autant, dans la communication de tous les jours, on continue de manière pragmatique de traduire Brot par pain, quand bien même l'horizon de sens de chacun de ces mots s'en trouve sensiblement « raboté ». Il va sans dire qu'il appartient au traducteur de décider quel horizon de sens il y a lieu de « raboter » et lequel mérite d'être mis en avant en fonction des exigences pragmatiques d'une traduction concrète. C'est la raison pour laquelle l'espace entre les langues est également un espace de recréation. Si en matière littéraire, on peut admettre que cette activité «(re)créatrice » prenne une ampleur plus importante, il nous semble qu'en matière de traduction pragmatique elle se trouve limitée par l'exigence de produire des textes satisfaisants [16, p. 51]. En définitive, pour délester le destinataire de la traduction du fastidieux travail d'élaborer un texte intelligible pour lui et pour réaliser la visée communicative de la traduction, il s'agit donc de produire des textes pertinents [13, p. 164]. Il en résulte deux conséquences majeures en termes de traduction interlinguistique : l'absence d'équipollence des interprétations et des choix de traduction sur le plan lexico-grammatical et stylistique d'une part, et d'autre part, le rôle majeur dévolu à la notion de pertinence, définie comme la sélection des éléments adéquats en termes de connaissances, de terminologie et de schémas lexico-grammaticaux.

Quelle que soit la caractérisation retenue de l'espace d'oscillation, la sémantique de l'espace implique nécessairement l'existence d'une frontière. La fonction de la frontière est double. En premier lieu, elle détermine les contours de l'espace singulier dans lequel se déploie un système symbolique et le définit ainsi comme

\footnotetext{
${ }^{23}$ S'agissant de ce terme, il semble que ce soit un régionalisme; dans d'autres régions de la Pologne, on utilisait bułka warszawska [pain varsovien], mais cela est sans importance pour notre propos.
} 
un système clos. Les frontières peuvent être externes ou internes. Si les frontières externes délimitent les aires linguistiques et culturelles ou les systèmes juridiques, les frontières internes tracent la limite de la compréhension intersubjective qui demeure une " une notion limite ", même si les conditions de communication sont optimales [37, p. 123]. Les frontières internes décrivent, p. ex. les limites entre la langue commune et une langue de spécialité. En second lieu, la frontière matérialise la clôture d'un espace et pose la pose la question de son éventuelle ouverture. La possibilité de l'ouverture de la frontière, même provisoire, conditionne la création de l'espace interstitiel et implique une certaine élasticité ou perméabilité de la frontière entre les espaces linguistiques, culturels et les systèmes juridiques.

Tout espace délimité par une frontière, et donc aussi tout espace symbolique tel qu'un univers sémantique, est un espace clos. En raison de cette clôture et de la finitude des univers sémantiques [35, p. 206], l'ouverture de la frontière et le passage entre eux [35, p. 209] semblent a priori compromis. Pourtant, l'espace interstitiel implique à titre de condition préalable au moins une certaine perméabilité, si ce n'est l'ouverture de la frontière entre les espaces symboliques. Pour appréhender cette nécessaire ouverture, on peut recourir à la notion de clôture opératoire ${ }^{24}$. Elle se définit comme « une limite (...) produite et reproduite dans tout évènement de communication spécifique et dont la fonction consiste à permettre l'ouverture et la fermeture d'un système » $[48$, p. 146]. La frontière entendue comme clôture opératoire n'est ni figée ni imperméable. Au contraire, elle possède une plasticité qui permet de l'ouvrir et la refermer au gré des besoins de communication. Ainsi, toute traduction concrète en tant qu'acte de communication ouvre la frontière entre les espaces linguistiques et culturels et la referme dès que la traduction est réalisée. L'ouverture de la clôture opératoire entre les univers sémantiques a pour effet la création de l'espace interstitiel dans lequel ont lieu les processus de la transdifférence permettant la constitution du sens dans l'intégration des sémantiques distinctes en jeu. Cependant, l'ouverture de la clôture opératoire entre les espaces symboliques ne se produit pas spontanément. Par conséquent, il se pose la question du mécanisme qui permet d'amorcer cette ouverture.

\subsection{L'intégration de l'altérité en traduction : la pertinence comme opérateur de l'ouverture de la clôture opératoire entre les univers sémantiques et l'appropriation réflexive}

Pour caractériser le mécanisme permettant d'amorcer l'ouverture de la clôture opératoire entre les langues dans le processus de la traduction interlinguistique, il est proposé ici de recourir à la notion de pertinence d'extraction non mentaliste, théorisée en sciences sociales par Alfred Schütz et opérationnalisée récemment pour les besoins de la traduction juridique [13]. En ce qui concerne la traduction interlinguistique, la notion de pertinence non mentaliste est investie d'une valeur heuristique élevée, car elle permet de rendre compte de différents aspects de la traduction, à

\footnotetext{
24 Traduction du terme allemand operative Schliessung, forgé par Niklas Luhmann dans sa théorie des systèmes.
} 
savoir la compréhension du texte, la constitution du sens, le rôle de l'interprétation et le mécanisme qui commande le choix des solutions de traduction. Enfin, l'adéquation du texte traduit à l'univers sémantique dans lequel il est censé s'inscrire peut également être caractérisée en termes de pertinence, définie comme propriété émergente du texte traduit. Les différentes définitions schützéennes de la pertinence, loin de constituer une tare, soulignent son caractère dynamique et mettent en exergue ses différents aspects mentionnés ci-dessus. Ainsi, la pertinence a été définie comme la relation dans le processus de compréhension [38, p. 47], comme le corrélat du sens [38, p. 49] et, enfin, comme le mécanisme de la sélection qui préside à l'élaboration du sens dans l'interprétation [38, p. 51]. L'intérêt majeur de la notion de pertinence pour la traduction réside dans la mise en exergue du lien fondamental entre le sens et la pertinence, d'une part, et d'autre part, de la nature relationnelle de celle-ci.

En ce qui concerne la traduction au sens classique du terme, il nous semble judicieux de souligner que la pertinence en tant que modus operandi de la sélection est apte à mettre un terme à la sémiose et à fixer concrètement le sens d'un texte traduit. Elle permet, en effet, la discrimination fine des schémas lexicogrammaticaux en fonction d'une traduction concrète. En outre, elle commande le choix des connaissances et des éléments à retenir pour une interprétation et lui confère ainsi de la légitimité. La pertinence étant le corrélat du sens, elle participe à la création des « contextes de sens » [38, p. 49]. Dans le processus de traduction, la pertinence se manifeste à la fois sur le plan micro- et macrotextuel. Sur le plan microtextuel ou local, elle opère en tant que principe organisateur de la sélection et détermine le choix des unités sémantiques, des constructions syntaxiques retenues et le style à adopter par le traducteur. Sur le plan macrotextuel ou global, la pertinence détermine l'efficacité de la traduction en tant qu'acte de communication. C'est la raison pour laquelle la pertinence peut être qualifiée de propriété émergente du texte traduit. Elle se définit comme une nouvelle propriété issue de l'interaction récursive et réciproque entre les phrases, les mots et les parties du texte [13, p. 298]. La pertinence comme propriété émergente de la traduction permet d'articuler le rapport de codétermination qui s'établit dans le processus de la traduction entre l'objet des connaissances et le sujet connaissant [50, p. 92; 20]. En d'autres termes, elle explique le rapport de spécification mutuelle entre le traducteur et les textes source et cible. Cette codétermination fait partie intégrante de la resémantisation au sein de l'espace interstitiel entre les univers linguistiques et culturels. Les processus sélectifs qui interviennent dans la traduction sous la rection de la pertinence permettent l'ouverture de la clôture opératoire entre les univers sémantiques et, corrélativement, la création de l'espace interstitiel. Le degré d'ouverture de la clôture opératoire des systèmes dépend de ce que Luhmann a appelé l'Anschlussfähigkeit. Il s'agit de la capacité d'un système à établir des contacts, p. ex. dans la communication, grâce à des éléments nouveaux ou utiles dans une situation concrète. Cette notion peut être traduite librement par « l'aptitude à s'arrimer » à un dispositif épistémologique. Un texte susceptible de s'insérer dans un dispositif épistémologique donné est utile, car il apporte au lecteur des informations ou des connaissances nouvelles [45]. Considéré de ce point de vue, le degré d'ouverture de la clôture opératoire dans la 
traduction interlinguale dépend donc de la capacité du traducteur d'arrimer le texte source grâce aux choix lexico-grammaticaux et stylistiques qu'il opère à un dispositif épistémologique intelligible pour le destinataire de la traduction. En définitive, le degré de l'ouverture de la clôture opératoire entre les espaces linguistiques dépend donc de la capacité du traducteur à produire un texte pertinent. On peut l'illustrer en reprenant l'exemple cité ci-dessus de la Zugewinngemeinschaft. En fonction de l'information recherchée, la traduction de ce terme en français variera. Ainsi, p. ex., si la traduction est destinée à un magistrat qui souhaite savoir uniquement s'il s'agit d'un régime de la communauté ou de la séparation de biens, on pourrait opter pour la traduction suivante : « le régime matrimonial de droit commun qui est un régime de la séparation de biens ». En revanche, si le destinataire de la traduction cherche à connaître précisément le contenu de ce régime matrimonial, on pourrait opter pour une traduction assortie d'une explicitation : "le régime légal, dit de la communauté de biens réduite aux acquêts qui, dans les faits, est un régime de la séparation de biens ». Les deux traductions sont pertinentes, leur pertinence étant déterminée par les exigences pragmatiques de la situation de communication. Pour résumer, on peut dire qu'en tant qu'opérateur de l'ouverture de la clôture opératoire entre les univers sémantiques, la pertinence est constitutive de l'intervalle, c'est-à-dire de l'espace interstitiel créé entre les différents univers sémantiques dans le processus de la traduction. Par-delà les différents modes d'organisation de la pensée et les différentes visions du monde dont les langues sont constitutives, la pertinence est apte à ouvrir un horizon commun permettant d'intégrer les sémantiques contradictoires et les différences culturelles qui en résultent [47, p. 263]. Cet horizon partagé participe de la compréhension réciproque et délimite l'espace partagé de communication [36, p. 151].

Si la traduction est apte à inscrire les éléments de l'altérité dans un horizon commun et à créer un consensus, même minimal et provisoire, c'est parce qu'elle ne se réduit pas simplement à l'immersion dans l'altérité, mais suppose une intégration des éléments étrangers (fremd) ou différents (anderes) d'un texte rédigé dans une langue étrangère sans pour autant tomber dans le piège du littéralisme Comme mentionné précédemment, l'intégration visée dans la traduction concerne la forme de la pensée, la forme de l'action et la forme linguistique [44, p. 142]. Elle doit s'opérer donc sur trois plans différents, à savoir sur le plan de la cognition, de la pratique et de l'expression. L'intégration de l'altérité ainsi comprise nécessite, au-delà de la compétence linguistique, la maîtrise des archives qui déterminent la sémantique du texte à traduire [44, p. 158] et qui permettent de l'inscrire dans le contexte pragmatique adéquat. Elle s'effectue dans les actes d'appropriation réflexive et sélective [44, p. 157] qui consistent à reconstruire les éléments de la sémantique étrangère tout en leur adjoignant des éléments familiers [44, p. 174]. L'appropriation réflexive, qui se déroule dans l'espace de réflexivité caractérisé ci-dessus comme un « entre-deux », permet la mise en relation de deux univers de sens distincts mis en jeu dans la traduction. Elle constitue donc une stratégie de gestion de la transdifférence et de la distance culturelle. Le texte traduit issu des actes d'appropriation réflexive et sélective effectués par le traducteur constitue un objet construit qui s'enracine dans les deux univers sémantiques à la fois. Par conséquent, elle représente un objet hybride tel que nous l'avons 
caractérisé sous le point 3.1. La mise en œuvre de la sélection, caractérisée cidessus comme le mécanisme de la pertinence, et la codétermination entre le traducteur et les textes source et cible impliquent que la traduction est un processus triadique dans lequel le discours étranger est resémantisé dans un autre système de signes par le biais de l'appropriation réflexive effectuée dans la subjectivité du traducteur. Cette resémantisation intervient toujours par rapport au monde de la vie quotidienne (Alltagswelt) [35, p. 148] qui constitue le tertium comparationis présupposé de la traduction. La mise en exergue de la nature réflexive du processus de l'appropriation permet d'écarter le reproche d'ethnocentrisme qui pourrait être formulé à l'égard de cette conception de la traduction interlinguistique.

Ainsi, et contrairement à la traduction culturelle au sens de Bhabha, qui conduit à l'affrontement de deux points de vue antagonistes et à une fragmentation du discours, la traduction interlinguistique vise à inscrire l'altérité dans un horizon partagé, et donc, à l'intégrer. Dès lors, elle constitue le lieu d'intégration de l'altérité sans pour autant dissoudre sa spécificité dans une appropriation ethnocentrique excessive ni l'exacerber dans un excès d'étrangeté et de littéralisme. La mise en œuvre de la sélection dans les actes de l'appropriation réflexive permet de résoudre les difficultés pratiques que soulève la gestion de la différence culturelle en traduction.

\section{Conclusion}

Pour conclure, et répondre à la question posée dans le titre, il nous semble que l'articulation des notions de traduction culturelle, d'hybridité et de tiers-espace conceptualisées par Bhabha en traduction pragmatique, et notamment juridique, se heurte à une difficulté tant sur le plan théorique que sur le plan pratique.

Sur le plan théorique, il est toujours possible d'élaborer des constructions permettant le transfert d'une notion d'un champ disciplinaire à un autre. Réussir un tel transfert implique néanmoins une reconceptualisation de la notion en question et, corrélativement, les modifications de sa signification qui doivent être précisées. En d'autres termes, tout transfert nécessite de redéfinir la notion et de déterminer son champ d'application. Par ailleurs, en ce qui concerne la traduction interlinguistique, les outils déjà existants, mobilisés dans le présent article, fournissent un cadre théorique et méthodologique permettant d'appréhender les processus complexes d'interaction réciproque à l'œuvre dans la traduction et les modalités d'intégration des éléments de l'altérité. Qui plus est, ces outils semblent mieux à même de rendre compte de ces processus, car ils permettent de mettre un terme à la différenciation du sens à l'infini et d'appréhender ces processus à partir d'une perspective plus large que la perspective d'un individu en marge d'une société.

Sur le plan pratique, la difficulté majeure concerne l'articulation de la théorie de la traduction culturelle de Bhabha à la pratique de la traduction juridique. En raison des contours flous et de la nature diffuse des notions de traduction culturelles, d'hybridité et de tiers-espace, il semble difficile de déterminer les modalités de leur 
intégration dans la pratique de la traduction interlinguistique, notamment juridique. Comme l'illustrent les exemples pratiques cités, le problème majeur de la traduction interlinguistique consiste à gérer la distance culturelle pour permettre une communication efficace entre les acteurs qui ne partagent ni la langue ni les cadres de référence culturels. Or la théorie de Bhabha n'apporte pas de réponse concrète à cette question.

Author contributions Dans la présente contribution, nous proposons d'explorer les différents cadres méthodologiques afin de construire une articulation de la notion de tiers-espace susceptible de rendre compte de la traduction au sens linguistique du terme.

Funding Not applicable for that section.

Data availability Not applicable for that section.

Code availability Not applicable for that section.

\section{Compliance with ethical standards}

Conflict of interest The authors declare that they have no conflict of interest.

Open Access This article is licensed under a Creative Commons Attribution 4.0 International License, which permits use, sharing, adaptation, distribution and reproduction in any medium or format, as long as you give appropriate credit to the original author(s) and the source, provide a link to the Creative Commons licence, and indicate if changes were made. The images or other third party material in this article are included in the article's Creative Commons licence, unless indicated otherwise in a credit line to the material. If material is not included in the article's Creative Commons licence and your intended use is not permitted by statutory regulation or exceeds the permitted use, you will need to obtain permission directly from the copyright holder. To view a copy of this licence, visit http://creativecommons.org/licen ses/by/4.0/.

\section{References}

1. Anfeng, Sheng, and Homi K. Bhabha. 2009. Minoritization as a global measure in the age of global postcoloniality: An interview with Homi K. Bhabha. The Free Library 1 (40): 161-180.

2. Bachmann-Medick, Doris. 2009. Introduction: The translational turn. Translation Studies 1 (2): 2-16. https://doi.org/10.1080/14781700802496118.

3. Bachmann-Medick, Doris. 2010-2013. Translational turn. In Handbook of translation studies, vol. 4, ed. Yves Gambier and Luc van Doorslaer, 186-193. Handbook of translation studies. Amsterdam: J. Benjamins.

4. Bakhtine, Mikhail Mikhaillovitch. 1987. Esthétique et théorie du roman. Collection Tel. Paris: Gallimard.

5. Bal, Mieke. 2002. Travelling concepts in the humanities. A rough guide. Green College lecture series. Toronto, Buffalo, London: University of Toronto press.

6. Bayart, Jean-François. 2010. Les Études postcoloniales. Un carnaval académique. Politique étrangère Hiver(4): 912. https://doi.org/10.3917/pe.104.0912.

7. Bhabha, Homi K. 2007. Les lieux de la culture. Une théorie postcoloniale. Paris: Payot.

8. Bhabha, Homi K., and Jonathan Rutherford. 2006. Le tiers-espace. Multitudes 26(3): 95. https://doi. org/10.3917/mult.026.0095.

9. Cao, Deborah. 2007. Translating law. Topics in translation, vol. 1. Clevendon: Multilingual Matters. 
10. Cassin, Barbara. 2016. Éloge de la traduction. Compliquer l'universel. Ouvertures, vol. 1. Paris: Fayard.

11. Chalard-Fillaudeau, Anne, and Gérard Raulet. 2003. Pour une critique des sciences de la culture. L'Homme et la société (Paris) 3 (149): 3-30.

12. Depraz, Natalie, Francisco J. Varela, and Pierre Vermersch. 2011. À l'épreuve de l'expérience. Pour une pratique phénoménologique. Phenomenological workshop texts, vol. 1. Bucarest: Zeta Books.

13. Durr, Margarete. 2020. La pertinence en traduction juridique. Un regard franco-allemand. Contrastes. Berlin, Bern, Bruxelles, New York, Oxford, Warszawa, Wien: Peter Lang.

14. Eco, Umberto. 2006. Dire presque la même chose. Paris: Grasset.

15. Felder, Ekkehard. 2008. Semantische Kämpfe in Wissensdomänen: Eine Einführung in Benennungs- Bedeutungs- und Sachverhaltsfixierungs-Konkurrenzen. In Semantische Kämpfe: Macht und Sprache in den Wissenschaften, ed. Ekkehard Felder, 353-371. Linguistik - Impulse \& Tendenzen, vol. 19. Berlin, New York: Walter de Gruyter.

16. Froeliger, Nicolas. 2013. Les noces de l'analogique et du numérique. De la traduction pragmatique. Traductologiques, vol. 1. Paris: Les Belles Lettres.

17. Frydman, Benoît. 2011. Le sens des lois. Histoire de l'interprétation et de la raison juridique, 3rd edn. Penser le droit, vol. 14. Bruxelles: Bruylant.

18. Galdia, Marcus. 2017. Lectures on legal linguistics. Frankfurt am Main: Peter Lang Edition.

19. Gledhill, Christopher, and Natalie Kübler. 2016. What can linguistic approaches bring to English for specific purposes? ASp. la revue du GERAS 69: 65-95. https://doi.org/10.4000/asp.4804.

20. Greisdorf, Howard. 2000. Relevance: An interdisciplinary and information science perspective. Informing Science 2: 3 .

21. Halliday, Michael. 1975. Learning how to mean: Explorations in the development of language. Londres: Edward Arnold.

22. Jacoby, Florian, Michael von Hinden, and Jan Kropholler. 2020. Bürgerliches Gesetzbuch. Studienkommentar, 17th ed. München: C.H.Beck.

23. Jakobson, Roman. 1959. On linguistics aspects of translation. In On translation, ed. Reuben Arther Brower, 232-239. Cambridge, MA: Harvard University Press.

24. Jia, Hongwei. 2017. Roman Jakobson's triadic division of translation revisited. Chinese Semiotic Studies 1: 13. https://doi.org/10.1515/css-2017-0003.

25. Ladmiral, Jean-René. 2010. La traduction, phénomène interculturel et psychorelationnel. Meta 4 (55): 626-641.

26. Ladmiral, Jean-René, and Edmond Marc Lipiansky. 2015. La communication interculturelle. Paris: Les Belles Lettres.

27. Lotman, Yuri, M. 2000. Semiosfera. Saint Petersbourg: Iskusstvo.

28. Luther, Martin. 2017. Écrits sur la traduction. Traductologiques. Paris: Les Belles Lettres.

29. Moréteau, Olivier. 2009. Les frontières de la langue et du droit : vers une méthodologie de la traduction juridique. Revue internationale de droit comparé 4 (61): 695-713. https://doi.org/10.3406/ ridc.2009.19911.

30. Müller-Funk, Wolfgang. 2016. Theorien des Fremden. Eine Einführung. Utb, vol. 4569. Tübingen: UTB; Francke, A.

31. Ost, François. 2009. Le droit comme traduction. Mercure du Nord. Quebec: Presses de L'Université Laval.

32. Ost, François. 2009. Traduire. Défense et illustration du multilinguisme. Ouvertures. Paris: Fayard.

33. Pratt, Mary Louise. 1992. Imperial eyes. Travel writing and transculturation. London, New York: Routledge.

34. Renn, Joachim. 2006. Übersetzungsverhältnisse. Perspektiven einer pragmatischen Gesellschaftstheorie. Weilerswist: Velbrück Wissenschaft.

35. Schütz, Alfred. 2003. Theorie der Lebenswelt 1. Zur pragmatischen Schichtung der Lebenswelt. Alfred Schütz Werkausagabe, V.1. Konstanz: UVK Verlagsgesellschaft.

36. Schütz, Alfred. 2003. Theorie der Lebenswelt 2. Die kommunikative Ordnung der Lebenswelt. Alfred Schütz Werkausagabe, V.2. Konstanz: UVK Verlagsgesellschaft.

37. Schütz, Alfred. 2004. Der sinnhafte Aufbau der sozialen Welt. Eine Einleitung in die verstehende Soziologie, II. Konstanz: UVK Verlagsgesellschaft.

38. Schütz, Alfred. 2004. Relevanz und Handeln 1. Zur Phänomenologie des Alltagswissens. Werkausgabe/Alfred Schütz Bd 6 Relevanz und Handeln, VI.1. Konstanz: UVK Verlagsgesellschaft.

39. Schütz, Alfred. 2010. Zur Methodologie der Sozialwissenschaften. Alfred Schütz Werkausabe, IV. Konstanz: UVK Verlagsgesellschaft. 
40. Schütz, Alfred. 2011. Relevanz und Handeln 2. Gesellschaftliches Wissen und politisches Handeln. Alfred Schütz Werkausgabe, VI.2. Konstanz: UVK Verlagsgesellschaft.

41. Senn, Fritz. 1983. Die fruchtbare Illusion der Übersetzbarkeit. In Nichts gegen Joyce, Aufsätze 1959-1983, ed. Franz Cavigelli, 246-261. Zürich: Haffmann.

42. Siever, Holger. 2010. Übersetzen und Interpretation. Die Herausbildung der Übersetzungswissenschaft als eigenständige wissenschaftliche Disziplin im deutschen Sprachraum von 1960 bis 2000. Leipziger Studien zur angewandten Linguistik und Translatologie, vol. 8. Frankfurt am Main, New York: P. Lang.

43. Simon, Sherry. 2010-2013. Hybridity and translation. In Handbook of translation studies, vol. 2, ed. Yves Gambier and Luc van Doorslaer, 49-53. Handbook of translation studies. Amsterdam: J. Benjamins.

44. Srubar, Ilja. 2009. Kultur und Semantik. Wiesbaden: VS Verlag für Sozialwissenschaften; VS, Verl. für Sozialwissenschaften.

45. Stegmaier, Werner. 2008. Philosophie der Orientierung. Berlin: Walter de Gruyter.

46. Struve, Karen. 2013. Zur Aktualität von Homi K. Bhabha. Einleitung in sein Werk. Aktuelle und klassische Sozial- und Kulturwissenschaftler/innen. Wiesbaden: Springer Fachmedien Wiesbaden; Imprint; Springer VS.

47. Thouard, Denis. 2016. Et toute langue est étrangère. Le projet de Humboldt. Paris: Encre marine.

48. Tyulenev, Sergey. 2012. Applying Luhmann to translation studies. Translation in society, vol. 1. New York (N.Y.), London: Routledge.

49. Tyulenev, Sergey. 2014. Translation and Society. An introduction, vol. 1. London, New York: Routledge.

50. Varela, Francisco J. 1989. Connaître les sciences cognitives. Science ouverte. Paris: Éd. du Seuil.

51. Vieweg, Klaus. 2019. Hegel. Der Philosoph der Freiheit / Biographie. München: C.H. Beck.

52. Wagner, Anne. 2016. A space in-between-legal translation as a 'third space'. Journal of Civil Law Studies 1 (9): 167-190.

53. Wagner, Birgit. 2009. Kulturelle Übersetzung: Erkundungen über ein wanderndes Konzept. kakanien revisited.

54. Wagner, Birgit. 2017. Ein Muttermal, so schön wie ein Amberstückchen.: Das Verhältnis von sprachlicher und kultureller Übersetzung, diskutiert am Beispiel von Antoine Gallands Mille et une nuits. In Kultur und Übersetzung: Studien zu einem begrifflichen Verhältnis, ed. Lavinia Heller. Interkulturalität, Band 8. Bielefeld: Transcript.

55. Willaschek, Marcus, Jürgen Stolzenberg, Georg Mohr, and Stefano Bacin (eds.). 2017. Kant-lexikon. Studienausgabe. Berlin, Boston: De Gruyter.

56. Wismann, Heinz. 2014. Penser entre les langues. Champs. Essais. Paris: Flammarion.

57. Wittgenstein, Ludwig. 2004. Recherches philosophiques. Bibliothèque de philosophie. Paris: Gallimard.

58. Wolf, Michaela, and Alexandra Fukari (eds.). 2007. Constructing a sociology of translation. Benjamins translation library, vol. 1. Amsterdam, Philadelphia (Pa.): J. Benjamins.

59. Wolff, Leon. 2011. Legal Translation. In The Oxford handbook of translation studies, ed. Kirsten Malmkjaer and Kevin Windle, 228-242. Oxford: Oxford University Press.

60. Zakaria, Fareed, and Johan-Frédérik Hel-Guedj. 2011. Le monde post-américain. Collection Tempus, vol. 392. Paris: Perrin.

Publisher's Note Springer Nature remains neutral with regard to jurisdictional claims in published maps and institutional affiliations. 